\title{
Microblade-Based Societies in North China at the End of the Ice Age
}

\author{
Meng Zhang ${ }^{1,2}$ \\ 1 Department of Cultural Heritage and Museology, Fudan University, Shanghai 200433, China; \\ zhangmengwb@fudan.edu.cn \\ 2 Institute of Archaeological Science, Fudan University, Shanghai 200433, China
}

Received: 23 May 2020; Accepted: 3 July 2020; Published: 9 July 2020

\begin{abstract}
One of the most prominent cultural changes during the end of Ice Age in northeastern Asia was the adoption of microblade technology by prehistoric hunter-gatherers to deal with the challenge brought by the climate deterioration and oscillation during and post the Last Glacial Maximum (LGM). The Pleistocene to Holocene transition in North China witnessed the rise of broader spectrum subsistence alongside a series of cultural changes, including adoption of food production, highly mobile lifeways being replaced by sedentism, and the formation of new social organization based on their agricultural land-use patterns. From the perspective of technological change, this project aims to build a socio-ecological framework to examine the cultural change of prehistoric microblade-based societies. In contrast to previous studies, the present research employs a macroecological approach based on Binford's Constructing Frames of Reference (2001) to reconstruct the behaviors and demography of prehistoric foraging groups, under both modern and LGM climate conditions. Three case studies are conducted to show cultural and technological changes among microblade-based societies in North China during the Pleistocene-Holocene transition.
\end{abstract}

Keywords: microblade technology; broad spectrum revolution; Pleistocene to Holocene transition; origin of food production; hunter-gatherers; macroecology; Constructing Frames of Reference

\section{Introduction}

During the Pleistocene to Holocene transition, northern China not only witnessed a broad spectrum revolution, intensification, and food production, but also saw the increasing use of durable stone tools linked to a terrestrial plant-dependent economy and the fading of microblade technology linked to highly mobile hunting-dominated lifeways. This process is generalized by Chen and $\mathrm{Yu}[1,2]$ as the Paleolithic to Neolithic Transition (PNT) (ca. 15,000-8500 Carbon 14 [C14] BP) in the period after the Late Upper Paleolithic (LUP) (ca. 24,000-15,000 C14 BP). Archaeological records suggest that the early Neolithic sites and sites with cultivation dated to the terminal Pleistocene are both located in relatively small habitats in basins, valleys and foothills of forest-steppe ecotones in the zone between the terrestrial plant threshold and the storage threshold in Lewis Binford's system (effective temperature [ET] $=12.75-15.25^{\circ} \mathrm{C}$ ) [3]. Agriculture appeared on the North China Plain after humans achieved sophisticated food production technologies during the Middle Holocene. Chen also attributes food production to collectors who practiced logistical strategies in Binford's forager-collector system $[4,5]$. Microblade-based societies cannot securely be classified as either foragers or collectors, but according to Chen's proposition, it seems that the foragers in northern China (if there were such) may have changed their subsistence-settlement system to collecting during the terminal Pleistocene. Current archaeological records are inadequate to fully test this hypothesis, but they can be investigated from a macroecological and analogical viewpoint. Recent research indicates that in northern China during the transition from the unpacked to the packed population 
condition, hunting-dominated subsistence is expected to become gathering-dominated subsistence in the west (the Loess Plateau), and fishing-dominated subsistence in the east (North China Plain) in which lakes and marshes formed at the end of the Last Ice Age [6]. Most early sites associated with cultivation, ground stone tools, and microblade assemblages are located along the boundary of the gathering-fishing-dominated subsistence line, suggesting that prehistoric foraging societies might have been practicing a mixed economy.

This project only focuses on the relationship between the rise of food production and the decline of microblade-based societies, within the time range from the Last Glacial Maximum to early Holocene. The decline of microblade-based societies cannot be explained as humans migrating out to the frontiers, since the archaeological record in Northern China tends to support an explanation of cultural transition rather than replacement [7]. In the following three sub-topics concerning Northern China, I apply the macroecological approach to study the development and decline of microblade-based societies under the background of the PNT, with the time range from the LGM to the early Holocene.

\section{Methodology}

The present project employs an analogical research strategy from the anthropological side to evaluate ideas generalized from the archaeological side in the previous studies which mainly followed a cultural-historical paradigm. The main methodology adopted here is macroecology, developed from the late Lewis Binford's book Constructing Frames of Reference [3], which has been fully standardized by his colleague Amber Johnson as a calculation program EnvCalc 2.1 [8]. This program makes possible the reconstruction of prehistoric hunter-gatherers' foraging strategies possible on a global scale [9] and the regional scale $[7,10,11]$. More than 300 variables derived from the program can be grouped as four datasets: (1) Climate, biomes, and habitat; (2) Minimalist terrestrial model; (3) Modelling density-dependent change in hunter-gatherer subsistence; and (4) Growth rate model and density-controlled subsistence (the variables referring to the present research are listed in Table 1). These variables provide an irreplaceable referential framework to help anthropologists predict/project hunter-gatherers' foraging strategies in a specific region even the foraging populations no longer exist due to cultural transformation or resettlement. For example, several variables, including duration of growing season (GROWC), net aboveground productivity (NAGP), and terrestrial model value for population density (TERMD2), are used to test the hypotheses of the transition from hunter-gathering to farming in the Near East by Binford [3] (pp. 434-464).

Table 1. Key variables used in this research project.

\begin{tabular}{cccc}
\hline Topics and Models & Hunter-Gatherers & Variable & Definition $^{\mathbf{1}}$ \\
\hline & Climate & GROWC & $\begin{array}{c}\text { Length of growing season: Length of growing } \\
\text { season. Number of months with mean } \\
\text { temperatures greater than 8 degrees Celsius }\end{array}$ \\
\cline { 2 - 4 } $\begin{array}{c}\text { Climate, Biomes, } \\
\text { and Habitat }\end{array}$ & Biomes and habitat & ET & $\begin{array}{c}\text { Effective temperature: a measure designed to } \\
\text { examine biological implications of ambient } \\
\text { warmth }\end{array}$ \\
\cline { 2 - 4 } & BIO5 & NAGP & Net above-ground productivity \\
\cline { 2 - 4 } & EXPEY & $\begin{array}{c}\text { Expected moderate body-size ungulate } \\
\text { biomass }\left(\mathrm{kg} / \mathrm{km}^{2}\right)\end{array}$ \\
\hline
\end{tabular}


Table 1. Cont

\begin{tabular}{|c|c|c|c|}
\hline Topics and Models & Hunter-Gatherers & Variable & Definition $^{1}$ \\
\hline \multirow{4}{*}{$\begin{array}{c}\text { Minimalist } \\
\text { Terrestrial Model }\end{array}$} & \multirow{3}{*}{ Population density } & TERMH2 & $\begin{array}{l}\text { Number of persons per } 100 \mathrm{~km}^{2} \text { unit who } \\
\text { could be supported by the ungulate } \\
\text { resources alone }\end{array}$ \\
\hline & & TERMG2 & $\begin{array}{l}\text { Number of persons per } 100 \mathrm{~km}^{2} \text { unit who } \\
\text { could be supported by the plant } \\
\text { resources alone }\end{array}$ \\
\hline & & TERMD2 & $\begin{array}{c}\text { Population density expected at a particular } \\
\text { location, expressed in terms of persons } \\
\text { per } 100 \mathrm{~km}^{2}\end{array}$ \\
\hline & Subsistence specialty & SUBSPX2 & $\begin{array}{l}\text { Terrestrial model expected subsistence bias } \\
\text { for use with ethnographic cases } \\
\mathrm{h}=\text { hunting, } \mathrm{g}=\text { gathering, } \mathrm{m}=\text { mixed, } \\
\mathrm{u}=\text { uninhabited }\end{array}$ \\
\hline \multirow{7}{*}{$\begin{array}{c}\text { Modelling } \\
\text { Density-Dependent } \\
\text { Change in } \\
\text { Hunter-Gatherer } \\
\text { Subsistence }\end{array}$} & Subsistence & SUBSPE & $\begin{array}{c}\text { Ordinal classification of projected } \\
\text { hunter-gatherer subsistence specialty } \\
1=\text { hunting, } 2=\text { gathering, } 3=\text { fishing }\end{array}$ \\
\hline & Population density & WDEN & Projected hunter-gatherer density \\
\hline & \multirow{3}{*}{ Group size } & EXGRP1 & $\begin{array}{c}\text { Projected mean size of smallest residential } \\
\text { group, segmented by group pattern and } \\
\text { subsistence specialization bias }\end{array}$ \\
\hline & & EXGRP2 & $\begin{array}{c}\text { Projected mean size of largest residential } \\
\text { seasonal camps, segmented by group pattern } \\
\text { and subsistence specialization bias }\end{array}$ \\
\hline & & EXGRP3 & $\begin{array}{l}\text { Projected mean size of periodic regional } \\
\text { camps, segmented by group pattern and } \\
\text { subsistence specialization bias }\end{array}$ \\
\hline & \multirow{2}{*}{ Mobility } & EXDMOV1 & $\begin{array}{l}\text { Projected total distance moved, scaled for } \\
\text { subsistence type, for groups with year round } \\
\text { camp to camp mobility pattern }\end{array}$ \\
\hline & & EXDMOV2 & $\begin{array}{l}\text { Projected total distance moved, scaled for } \\
\text { subsistence type, for groups who move into } \\
\text { and out of a central location or who are } \\
\text { primarily sedentary }\end{array}$ \\
\hline \multirow{2}{*}{$\begin{array}{l}\text { Growth Rate Model } \\
\text { and } \\
\text { Density-Controlled } \\
\text { Subsistence }\end{array}$} & $\begin{array}{l}\text { Subsistence specialty } \\
\text { against the unpacked } \\
\text { background }\end{array}$ & UPSUBSPE & $\begin{array}{l}\text { Ordinal classification of projected unpacked } \\
\text { HG subsistence specialty } \\
\text { (packing multiplier }=0.5 \text { ) } \\
1=\text { hunting, } 2=\text { gathering, } 3=\text { fishing }\end{array}$ \\
\hline & $\begin{array}{l}\text { Subsistence specialty } \\
\text { against the packed } \\
\text { background }\end{array}$ & D1PSUBSPE & $\begin{array}{c}\text { Ordinal classification of projected packed HG } \\
\text { subsistence specialty (packing multiplier = 1) } \\
1=\text { hunting, } 2 \text { = gathering, } 3=\text { fishing }\end{array}$ \\
\hline
\end{tabular}

Note: ${ }^{1}$ from "variables used or calculated in EnvCalc2.1 program (except UPSUBSPE and D1PSUBSPE) [8]. 
The author's dissertation combines macroecological approach of hunter-gatherers, modern and simulated LGM climatic data, and prehistoric technological organization associated with microblade technology to investigate the role of microblade technology in the development of human adaptations in NE Asia, especially northern China, during the closing millennia of the Upper Pleistocene and across the Pleistocene-Holocene transition [6]. Microblade-based societies as a new concept provides a fresh perspective to characterize a socio-technologically adaptive convergence and radiation of human groups before and after the Last Glacial Maximum (LGM) across NE Asia, in which people adopted microblade technology as the basis for the lithic elements of composite tools/weapons to help organize their lives in their territories, depending, of course, on the availability and quality of local lithic resources.

\section{Archaeological Sites Associated with Microblade Technology in North China}

North China, in the narrow sense, refers two subregions: the Loess Plateau in the west and the North China Plain in the east. According to archaeological record recovered, the southern limit of distribution of microblade technology during the late Pleistocene is northern side of the Qinling Mountain and the Huaihe River, which is also the north-south division of modern Chinese geography. The northern line of North China is along the Great Wall, which is also the steppe-forest ecotone during the early Holocene.

Since this paper mainly focuses on the period of origin of food production, using Shizitan site as an example to discuss the possibility of plant-intensified exploitation behaviors during the LGM, and at the end, using the Donghulin site to talk about the demise of microblade-based societies, data collected for this project are associated with those sites dated to the Pleistocene-Holocene transition (Table 2). They are mainly Accelerator Mass Spectrometry Carbon 14 (AMS C14) dates, as well as latitudes and longitudes of the sites. To further discuss issues of the broad spectrum revolution, archaeological data are categorized into artifact assemblage, site organization, and biological remains are also shown in Table 3. 
Table 2. Chronometric dates of the sites in the Paleolithic to Neolithic Transition (PNT) group.

\begin{tabular}{|c|c|c|c|c|c|c|c|}
\hline Site & Layer & Material & Method & Lab. No. & Dates & Cal. BC (2-sigma) & Reference \\
\hline \multirow{7}{*}{ Nanzhuangtou } & layer 5-6 & wood & AMS C14 & BK86120 & $9875 \pm 160$ & $\begin{array}{c}10,028-9112(87.2 \%) \\
9083-9041(1.1 \%) \\
9026-8838(7.1 \%)\end{array}$ & Yuan et al. [12] \\
\hline & & wood & AMS C14 & Bk86121 & $9690 \pm 95$ & $9298-8799(95.4 \%)$ & Yuan et al. [12] \\
\hline & & wood & AMS C14 & BK87093 & $9810 \pm 100$ & $\begin{array}{c}9669-9116(86.1 \%) \\
9076-9055(0.6 \%) \\
9016-8843(8.7 \%)\end{array}$ & Yuan et al. [12] \\
\hline & & wood & AMS C14 & BK89064 & $9850 \pm 90$ & $\begin{array}{c}9752-9722(1 \%) \\
9692-9146(94.4 \%)\end{array}$ & Yuan et al. [12] \\
\hline & Layer 6 & charcoal & AMS C14 & BK87075 & $10,510 \pm 100$ & $10,731-10,149(95.4 \%)$ & Yuan et al. [12] \\
\hline & Layer 5 & silt & AMS C14 & BK87086 & $9980 \pm 100$ & $\begin{array}{c}9983-9941(1.6 \%) \\
9877-9266(93.8 \%)\end{array}$ & Yuan et al. [12] \\
\hline & & silt & AMS C14 & BK87088 & $10,815 \pm 140$ & $\begin{array}{r}11,096-10,576(94 \%) \\
10,518-10,485(1.4 \%)\end{array}$ & Yuan et al. [12] \\
\hline \multirow{2}{*}{ Zhuannian } & & & AMS C14 & & $9200 \pm 100$ & $\begin{array}{l}8701-8676(1.4 \%) \\
8646-8250(94 \%)\end{array}$ & Ren and $\mathrm{Wu}$ [13] \\
\hline & & & AMS C14 & & c.9800 & & Yu [14] \\
\hline \multirow{5}{*}{ Donghulin } & M1 (Burial 1) & human bone & AMS C14 & & $9570 \pm 70$ & $9216-8755(95.4 \%)$ & Cui [15] \\
\hline & Layer $8, \mathrm{~T} 3$ & charcoal & AMS C14 & & $9180 \pm 80$ & $8608-8273(95.4 \%)$ & Cui [15] \\
\hline & Layer 7, T3 & charcoal & AMS C14 & & $9155 \pm 40$ & $\begin{array}{c}8528-8521(0.8 \%) \\
8473-8282(94.6 \%)\end{array}$ & Cui [15] \\
\hline & Layer 4, T3 & charcoal & AMS C14 & & $8780 \pm 90$ & $\begin{array}{l}8205-8035(20.6 \%) \\
8016-7606(74.8 \%)\end{array}$ & Cui [15] \\
\hline & Layer 4, T9 & charcoal & AMS C14 & & $8805 \pm 50$ & $\begin{array}{c}8204-8102(13.8 \%) \\
8096-8036(5.6 \%) \\
8015-7711(75.6 \%) \\
7692-7685(0.4 \%)\end{array}$ & Cui [15] \\
\hline
\end{tabular}


Table 2. Cont

\begin{tabular}{|c|c|c|c|c|c|c|c|}
\hline Site & Layer & Material & Method & Lab. No. & Dates & Cal. BC (2-sigma) & Reference \\
\hline \multirow[t]{2}{*}{ Donghulin } & Layer 3, T9 & charcoal & AMS C14 & & $8772 \pm 40$ & $\begin{array}{c}8165-8138(1.5 \%) \\
7973-7647(93.9 \%)\end{array}$ & Cui [15] \\
\hline & Layer 2, T9 & Pineapple & AMS C14 & & $8885 \pm 55$ & $8240-7825(95.4 \%)$ & Cui [15] \\
\hline \multirow{3}{*}{ Lijiagou (north area) } & Layer 6 & charcoal & AMS C14 & BA091494 & $8950 \pm 40$ & $\begin{array}{l}8272-8166(44.3 \%) \\
8131-7970(51.1 \%)\end{array}$ & He et al. [16] \\
\hline & Layer 5 & charcoal & AMS C14 & BA091417 & $8015 \pm 35$ & 7064-6814 (95.4\%) & He et al. [16] \\
\hline & Layer 4 & charcoal & AMS C14 & BA091416 & $7740 \pm 40$ & $6642-6483(95.4 \%)$ & He et al. [16] \\
\hline Lijiagou (south area) & Layer 4 & $\begin{array}{l}\text { charcoal (3 } \\
\text { samples) }\end{array}$ & AMS C14 & \multicolumn{2}{|c|}{ 10300-10500 cal. BP } & & Zhao et al. [17] \\
\hline \multirow{9}{*}{ Bianbiandong } & & Human skull & AMS C14 & BA04308 & $8675 \pm 40$ & $\begin{array}{c}7786-7768(2 \%) \\
7759-7592(93.4 \%)\end{array}$ & Sun et al. [18] \\
\hline & & Human skull & AMS C14 & BA04309 & $8670 \pm 30$ & $7738-7597(95.4 \%)$ & Sun et al. [18] \\
\hline & & Animal skull & AMS C14 & BA04310 & $10,030 \pm 40$ & $\begin{array}{c}9800-9786(1.2 \%) \\
9773-9381(94.2 \%)\end{array}$ & Sun et al. [18] \\
\hline & & Human skull & AMS C14 & BA04317 & $8730 \pm 50$ & $7941-7605(95.4 \%)$ & Sun et al. [18] \\
\hline & Area I Layer 3A & Animal bone & AMS C14 & BA07226 & $8505 \pm 45$ & 7596-7507 (95.4\%) & Sun et al. [18] \\
\hline & Area I Layer 3B & Animal bone & AMS C14 & BA07227 & $8585 \pm 40$ & $\begin{array}{c}7704-7700(0.5 \%) \\
7681-7541(94.9 \%)\end{array}$ & Sun et al. [18] \\
\hline & Area I Layer 4 & Animal bone & AMS C14 & BA07228 & $8180 \pm 45$ & $7321-7067(95.4 \%)$ & Sun et al. [18] \\
\hline & Layer 2 & Animal bone & AMS C14 & BA07229 & $6120 \pm 45$ & $5211-4944(95.4 \%)$ & Sun et al. [18] \\
\hline & Collected & Animal bone & AMS C14 & BA07230 & $6305 \pm 40$ & $5364-5214(95.4 \%)$ & Sun et al. [18] \\
\hline Kengnan & Layer 2, 3 & & C14 & \multicolumn{2}{|c|}{ 10,000-9000 BP (calibrated?) } & & Li, Song [19] \\
\hline Yujiagou & & & OSL & & 18 ka-14 ka & & Mei [20] \\
\hline Ma'anshan $^{1}$ & & & & & - & & \\
\hline
\end{tabular}


Table 2. Cont.

\begin{tabular}{|c|c|c|c|c|c|c|c|}
\hline Site & Layer & Material & Method & Lab. No. & Dates & Cal. BC (2-sigma) & Reference \\
\hline \multirow{3}{*}{ Hutouliang } & Terrace II & bone & Conv. C14 & PV-0156 & $10,690 \pm 120$ & $\begin{array}{c}10,879-10,428(93.3 \%) \\
10,391-10,352(0.8 \%) \\
10,335-10,287(1.2 \%)\end{array}$ & IA-CASS [21] \\
\hline & Layer 6 (upper) & polymineral & OSL & & $9.2 \pm 1.4 \mathrm{ka}$ & & Nagatomo et al. [22] \\
\hline & & polymineral & OSL & & $9.0 \pm 1.3 \mathrm{ka}$ & & Nagatomo et al. [22] \\
\hline Mengjiaquan & & & & & - & & \\
\hline Jijitan $^{1}$ & & & & & - & & \\
\hline Xueguan & & charcoal & Conv.C14 & BK81016 & $13,170 \pm 150$ & $14,274-13,371(95.4 \%)$ & IA-CASS [21] \\
\hline \multirow{12}{*}{ Lingjing } & Layer 5 & charred material & AMS C14 & IAAA-92123 & $11,360 \pm 50$ & $11,350-11,145$ (95.4\%) & Li et al. [23] \\
\hline & Layer 5 & charred material & AMS C14 & IAAA-92124 & $11,490 \pm 50$ & $11,502-11,285(95.4 \%)$ & Li et al. [23] \\
\hline & Layer 5 & charred material & AMS C14 & IAAA-92125 & $11,930 \pm 50$ & $\begin{array}{c}12,009-11,930(8.6 \%) \\
11,915-11,625(86.8 \%)\end{array}$ & Li et al. [23] \\
\hline & Layer 5 & charcoal & AMS C14 & IAAA-100080 & $28,610 \pm 120$ & $31,223-30,139(95.4 \%)$ & Li et al. [23] \\
\hline & Layer 5 & burnt bone & AMS C14 & IAAA-100082 & $11,520 \pm 40$ & 11,499-11,331 (95.4\%) & Li et al. [23] \\
\hline & Layer 5 & burnt bone & AMS C14 & IAAA-102634 & $11,400 \pm 50$ & $11,406-11,168$ (95.4\%) & Li et al. [23] \\
\hline & Layer 5 & charcoal & AMS C14 & IAAA-102635 & $11,600 \pm 50$ & $11,601-11,365(95.4 \%)$ & Li et al. [23] \\
\hline & Layer 5 & $\begin{array}{l}\text { charred material } \\
\text { on sherd }\end{array}$ & AMS C14 & IAAA-102636 & $8610 \pm 40$ & $7723-7576(95.4 \%)$ & Li et al. [23] \\
\hline & Layer 5 & $\begin{array}{l}\text { charred material } \\
\text { (charcoal?) }\end{array}$ & AMS C14 & IAAA-102638 & $10,180 \pm 40$ & $10,106-9757(95.4 \%)$ & Li et al. [23] \\
\hline & Layer 5 & $\begin{array}{l}\text { charred material } \\
\text { (charcoal?) }\end{array}$ & AMS C14 & IAAA-102639 & $11,710 \pm 50$ & $\begin{array}{c}11,763-11,721(3.2 \%) \\
11,681-11,483(92.2 \%)\end{array}$ & Li et al. [23] \\
\hline & Layer 5 & $\begin{array}{l}\text { charred material } \\
\text { (charcoal?) }\end{array}$ & AMS C14 & IAAA-102640 & $11,860 \pm 50$ & $11,822-11,613(95.4 \%)$ & Li et al. [23] \\
\hline & Layer 5 & burnt bone & AMS C14 & IAAA-102641 & $11,290 \pm 50$ & $11,307-11,115(95.4 \%)$ & Li et al. [23] \\
\hline
\end{tabular}


Table 2. Cont

\begin{tabular}{|c|c|c|c|c|c|c|c|}
\hline Site & Layer & Material & Method & Lab. No. & Dates & Cal. BC (2-sigma) & Reference \\
\hline \multirow{8}{*}{ Lingjing } & Layer 5 & $\begin{array}{l}\text { charred material } \\
\text { (charcoal?) }\end{array}$ & AMS C14 & IAAA-102642 & $10,970 \pm 50$ & $11,032-10,772(95.4 \%)$ & Li et al. [23] \\
\hline & Layer 5 & $\begin{array}{l}\text { charred material } \\
\text { (burnt bone?) }\end{array}$ & AMS C14 & IAAA-102643 & $11,280 \pm 50$ & $11,302-11,109$ (94.4\%) & Li et al. [23] \\
\hline & Layer 5 & Charcoal (?) & AMS C14 & IAAA-102644 & $11,550 \pm 50$ & $11,525-11,335$ (95.4\%) & Li et al. [23] \\
\hline & Layer 5 & $\begin{array}{c}\text { charred material } \\
\text { (?) }\end{array}$ & AMS C14 & IAAA-102645 & $11,470 \pm 50$ & $11,489-11,251$ (95.4\%) & Li et al. [23] \\
\hline & Layer 5 & charcoal & AMS C14 & IAAA-102647 & $11,220 \pm 50$ & $11,242-11,046(95.4 \%)$ & Li et al. [23] \\
\hline & Layer 5 & charcoal & AMS C14 & IAAA-102648 & $11,520 \pm 50$ & $11,511-11,321(95.4 \%)$ & Li et al. [23] \\
\hline & Layer 5 & $\begin{array}{l}\text { charred material } \\
\text { (burnt bone?) }\end{array}$ & AMS C14 & IAAA-102649 & $11,800 \pm 50$ & $\begin{array}{r}11,801-11,587(89 \%) \\
11,576-11,532(6.4 \%)\end{array}$ & Li et al. [23] \\
\hline & Layer 5 & $\begin{array}{l}\text { charred material } \\
\text { (charcoal?) }\end{array}$ & AMS C14 & IAAA-102650 & $11,860 \pm 50$ & $11,822-11,613(95.4 \%)$ & Li et al. [23] \\
\hline \multirow{8}{*}{ Shizitan, Locality S29 } & Layer 1 & bone & AMS C14 & BA10129 & $11,175 \pm 60$ & $11,203-10,903$ (95.4\%) & Song [24] \\
\hline & $\begin{array}{c}\text { Layer } 2(203 \mathrm{~cm} \\
\text { deep) }\end{array}$ & Bone & AMS C14 & BA101414 & $14,650 \pm 70$ & $16,077-15,674(95.4 \%)$ & Song and Shi [25] \\
\hline & $\begin{array}{c}\text { Layer } 2(200-206 \\
\text { cm deep) }\end{array}$ & Bone & AMS C14 & BA10132 & $15,725 \pm 80$ & $17,238-16,844(95.4 \%)$ & Song and Shi [25] \\
\hline & $\begin{array}{c}\text { Layer } 2(248 \mathrm{~cm} \\
\text { deep })\end{array}$ & Charcoal & AMS C14 & BA10131 & $16,760 \pm 65$ & $18,494-18,058$ (95.4\%) & Song and Shi [25] \\
\hline & $\begin{array}{c}\text { Layer } 2(276 \mathrm{~cm} \\
\text { deep })\end{array}$ & Bone & AMS C14 & BA101416 & $15,390 \pm 70$ & $16,862-16,553(95.4 \%)$ & Song and Shi [25] \\
\hline & $\begin{array}{c}\text { Layer } 3(282 \mathrm{~cm} \\
\text { deep) }\end{array}$ & Bone & AMS C14 & BA101419 & $17,200 \pm 50$ & $18,987-18,615$ (95.4\%) & Song and Shi [25] \\
\hline & $\begin{array}{c}\text { Layer } 3(303 \mathrm{~cm} \\
\text { deep) }\end{array}$ & Bone & AMS C14 & BA10133 & $17,360 \pm 60$ & $19,232-18,762(95.4 \%)$ & Song and Shi [25] \\
\hline & $\begin{array}{c}\text { Layer } 4(465 \mathrm{~cm} \\
\text { deep) }\end{array}$ & Bone & AMS C14 & BA101420 & $17,500 \pm 70$ & $19,449-18,936$ (95.4\%) & Song and Shi [25] \\
\hline
\end{tabular}


Table 2. Cont.

\begin{tabular}{|c|c|c|c|c|c|c|c|}
\hline Site & Layer & Material & Method & Lab. No. & Dates & Cal. BC (2-sigma) & Reference \\
\hline \multirow{14}{*}{ Shizitan, Locality S29 } & $\begin{array}{c}\text { Layer } 4(469 \mathrm{~cm} \\
\text { deep) }\end{array}$ & Bone & AMS C14 & BA10134 & $16,170 \pm 50$ & $17,754-17,367$ (95.4\%) & Song and Shi [25] \\
\hline & $\begin{array}{c}\text { Layer } 4(605 \mathrm{~cm} \\
\text { deep) }\end{array}$ & Bone & AMS C14 & BA10135 & $16,930 \pm 50$ & $18,649-18,268$ (95.4\%) & Song and Shi [25] \\
\hline & $\begin{array}{c}\text { Layer } 4(620 \mathrm{~cm} \\
\text { deep) }\end{array}$ & Bone & AMS C14 & BA101422 & $16,750 \pm 80$ & $18,511-18,030(95.4 \%)$ & Song and Shi [25] \\
\hline & $\begin{array}{c}\text { Layer } 4(622 \mathrm{~cm} \\
\text { deep) }\end{array}$ & Bone & AMS C14 & BA101421 & $18,570 \pm 60$ & $20,638-20,363(95.4 \%)$ & Song and Shi [25] \\
\hline & $\begin{array}{c}\text { Layer } 4(624 \mathrm{~cm} \\
\text { deep) }\end{array}$ & Bone & AMS C14 & BA101423 & $19,210 \pm 80$ & $21,495-20,943(95.4 \%)$ & Song and Shi [25] \\
\hline & $\begin{array}{c}\text { Layer } 4(640 \mathrm{~cm} \\
\text { deep) }\end{array}$ & Tooth & AMS C14 & BA10136 & $17,040 \pm 60$ & $18,804-18,391(95.4 \%)$ & Song and Shi [25] \\
\hline & $\begin{array}{c}\text { Layer } 5(772 \mathrm{~cm} \\
\text { deep) }\end{array}$ & Bone & AMS C14 & BA10137 & $18,360 \pm 70$ & $20,477-20,025(95.4 \%)$ & Song and Shi [25] \\
\hline & $\begin{array}{c}\text { Layer } 5(787 \mathrm{~cm} \\
\text { deep) }\end{array}$ & Charcoal & AMS C14 & BA10485 & $20,420 \pm 80$ & $22,973-22,319(95.4 \%)$ & Song and Shi [25] \\
\hline & $\begin{array}{l}\text { Layer } 5 \text { (750.5 } \\
\text { cm deep) }\end{array}$ & Bone & AMS C14 & BA101426 & $19,650 \pm 100$ & $22,028-21,432(95.4 \%)$ & Song and Shi [25] \\
\hline & $\begin{array}{l}\text { Layer } 5 \text { (751.5 } \\
\text { cm deep) }\end{array}$ & Charcoal & AMS C14 & BA101427 & $19,510 \pm 70$ & $21,815-21,244(95.4 \%)$ & Song and Shi [25] \\
\hline & $\begin{array}{l}\text { Layer } 5 \text { (750.5 } \\
\text { cm deep) }\end{array}$ & Charcoal & AMS C14 & BA101428 & $19,940 \pm 70$ & $22,284-21,811(95.4 \%)$ & Song and Shi [25] \\
\hline & $\begin{array}{c}\text { Layer } 5(804 \mathrm{~cm} \\
\text { deep) }\end{array}$ & Charcoal & AMS C14 & BA101429 & $19,710 \pm 80$ & $22,050-21,535(95.4 \%)$ & Song and Shi [25] \\
\hline & $\begin{array}{l}\text { Layer } 5 \text { (801.8 } \\
\text { cm deep) }\end{array}$ & Charcoal & AMS C14 & BA101430 & $19,860 \pm 70$ & $22,185-21,707(95.4 \%)$ & Song and Shi [25] \\
\hline & $\begin{array}{c}\text { Layer } 6(968 \mathrm{~cm} \\
\text { deep) }\end{array}$ & Bone & AMS C14 & BA101431 & $18,140 \pm 80$ & $20,326-19,812(95.4 \%)$ & Song and Shi [25] \\
\hline
\end{tabular}


Table 2. Cont.

\begin{tabular}{|c|c|c|c|c|c|c|c|}
\hline Site & Layer & Material & Method & Lab. No. & Dates & Cal. BC (2-sigma) & Reference \\
\hline \multirow{12}{*}{ Shizitan, Locality S29 } & $\begin{array}{c}\text { Layer } 6 \text { (964 cm } \\
\text { deep) }\end{array}$ & Charcoal & AMS C14 & BA101433 & $20,410 \pm 80$ & $22,961-22,306(95.4 \%)$ & Song and Shi [25] \\
\hline & $\begin{array}{c}\text { Layer } 6 \text { (964 cm } \\
\text { deep) }\end{array}$ & Bone & AMS C14 & BA101434 & $19,850 \pm 80$ & $22,190-21,680(95.4 \%)$ & Song and Shi [25] \\
\hline & $\begin{array}{l}\text { Layer } 6 \text { (961.5 } \\
\text { cm deep) }\end{array}$ & Tooth & AMS C14 & BA121954 & $20,155 \pm 45$ & $22,471-22,067$ (95.4\%) & Song and Shi [25] \\
\hline & $\begin{array}{c}\text { Layer } 6(1004 \mathrm{~cm} \\
\text { deep) }\end{array}$ & Charcoal & AMS C14 & BA10487 & $20,500 \pm 100$ & $23,117-22,388$ (95.4\%) & Song and Shi [25] \\
\hline & $\begin{array}{c}\text { Layer } 6(1004 \mathrm{~cm} \\
\text { deep) }\end{array}$ & Bone & AMS C14 & BA10488 & $18,090 \pm 70$ & $20,242-19,733(95.4 \%)$ & Song and Shi [25] \\
\hline & $\begin{array}{c}\text { Layer } 6(1004 \mathrm{~cm} \\
\text { deep) }\end{array}$ & Bone & AMS C14 & BA121951 & $18,280 \pm 45$ & 20,393-19,978 (95.4\%) & Song and Shi [25] \\
\hline & $\begin{array}{c}\text { Layer } 6(1026 \mathrm{~cm} \\
\text { deep) }\end{array}$ & Charcoal & AMS C14 & BA101438 & $20,350 \pm 90$ & $22,886-22,201(95.4 \%)$ & Song and Shi [25] \\
\hline & $\begin{array}{c}\text { Layer } 7(1160 \mathrm{~cm} \\
\text { deep) }\end{array}$ & Bone & AMS C14 & BA121960 & $21,690 \pm 80$ & $24,151-23,840(95.4 \%)$ & Song and Shi [25] \\
\hline & $\begin{array}{c}\text { Layer } 7(1160 \mathrm{~cm} \\
\text { deep) }\end{array}$ & Bone & AMS C14 & BA101439 & $19,650 \pm 80$ & $21,996-21,465(95.4 \%)$ & Song and Shi [25] \\
\hline & $\begin{array}{c}\text { Layer } 7(1160 \mathrm{~cm} \\
\text { deep })\end{array}$ & Charcoal & AMS C14 & BA101442 & $20,010 \pm 70$ & $22,353-21,901(95.4 \%)$ & Song and Shi [25] \\
\hline & $\begin{array}{c}\text { Layer } 7-8 \\
\text { boundary }(1355 \\
\text { cm deep) }\end{array}$ & Bone & AMS C14 & BA101445 & $20,510 \pm 90$ & $23,107-22,414(95.4 \%)$ & Song and Shi [25] \\
\hline & $\begin{array}{c}\text { Layer } 8(1425 \mathrm{~cm} \\
\text { deep) }\end{array}$ & Charcoal & AMS C14 & BA101444 & $24,185 \pm 90$ & $26,575-25,976(95.4 \%)$ & Song and Shi [25] \\
\hline Shizitan, Locality S5 & Layer 1 & bone & AMS C14 & BA101404 & $9220 \pm 50$ & $8565-8299(95.4 \%)$ & Song and Shi [26] \\
\hline
\end{tabular}


Table 2. Cont.

\begin{tabular}{|c|c|c|c|c|c|c|c|}
\hline Site & Layer & Material & Method & Lab. No. & Dates & Cal. BC (2-sigma) & Reference \\
\hline \multicolumn{8}{|c|}{ Some other sites dated to PNT time range, but not related to origin of food production (data shown below, recorded as others in the maps) } \\
\hline \multirow{8}{*}{$\begin{array}{l}\text { Shuidonggou } \\
\text { Locality } 12\end{array}$} & & & & & & $9654-9580(2.5 \%)$ & \\
\hline & & & & & & $9548-9478(2.3 \%)$ & \\
\hline & Laver 11 & charcoal & AMS C14 & LUG06-54 & $9797+91$ & 9465-9117 (80.9\%) & Liu et al. [27] \\
\hline & Layer 11 & Charcod & Alvis 14 & LU & 9798 & 9074-9056 (0.5\%) & Liu et ail. $[27]$ \\
\hline & & & & & & 9013-8911 (7\%) & \\
\hline & & & & & & $8905-8845(2.2 \%)$ & \\
\hline & Layer 11 & quartz & OSL & IEE1110 & $11.6 \pm 0.6 \mathrm{ka}$ & & Nagatomo et al. [22] \\
\hline & L1-L5 & cultural layers & $\begin{array}{l}\text { between } 11 \\
\text { middle of } t\end{array}$ & $\begin{array}{l}\text { and } 12,000 \text { year } \\
\text { unger Dryas }\end{array}$ & go, starting at & & Yi [28] \\
\hline \multirow{3}{*}{ Gezishan 53S/0W } & Stratum E & - & & Beta 97241 & $10,230 \pm 50$ & 10,189-9810 (95.4\%) & Elston et al. [29] \\
\hline & Stratum E/F & - & & Beta 86731 & $11,620 \pm 70$ & $11,626-11,353(95.4 \%)$ & Elston et al. [29] \\
\hline & Stratum G2 & - & & Beta 97242 & $12,710 \pm 70$ & $13,398-12,857$ (95.4\%) & Elston et al. [29] \\
\hline \multirow{2}{*}{ Gezishan 3N/3W } & Stratum D & - & & Beta 86732 & $10,020 \pm 60$ & 9818-9318 (95.4\%) & Elston et al. [29] \\
\hline & Stratum D & - & & Beta 97346 & $10,130 \pm 70$ & 10,086-9452 (95.4\%) & Elston et al. [29] \\
\hline PY-04 & & charcoal & AMS C14 & CAMS94202 & $10,670 \pm 40$ & $10,759-10,618$ (95.4\%) & Barton et al. [30] \\
\hline
\end{tabular}


Table 3. Presence of markers of the PNT in North China, by site ${ }^{3}$ Table 2 modified from [2]. These sites are mapped in Figure 1.

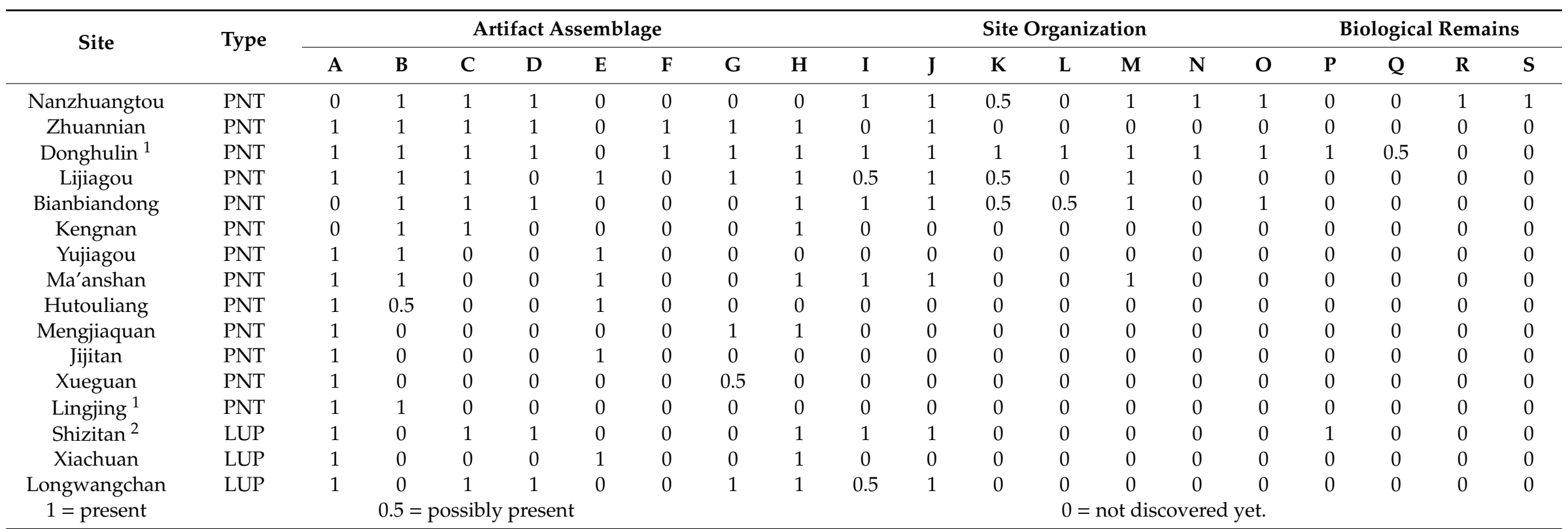

Notes: The rows: $\mathrm{A}=$ microblade, $\mathrm{B}=$ pottery, $\mathrm{C}=$ mortar (millstone), $\mathrm{D}=$ pestle (millstick), $\mathrm{E}=$ adze, $\mathrm{F}=$ stone container, $\mathrm{G}=$ polished tool, $\mathrm{H}=$ diversity of artifact types, $\mathrm{I}=$ hearth,

$\mathrm{J}=$ diversity of site types, $\mathrm{K}=$ structure, $\mathrm{L}=$ grave, $\mathrm{M}=$ central camp, $\mathrm{N}=$ ditch, $\mathrm{O}=$ pit, $\mathrm{P}=$ foxtail millet, $\mathrm{Q}=$ broomcorn millet, $\mathrm{R}=\operatorname{dog}$, $\mathrm{S}=$ wild pig (boar). The columns: Other sites without data except microblades: Xishi (Late Upper Paleolithic, LUP); Shuidonggou Loc. 12; Dadiwan (Component 4); Gezishan (Pigeon Mountain); PY-04, all classified as "other" (see maps of Northern Chinanorthern China). ${ }^{1}$ updated information according to Cui [15]; ${ }^{2}$ new site added according to Li et al. [23]; ${ }^{3}$ updated information of Locality 29 according to Liu et al. [31] 


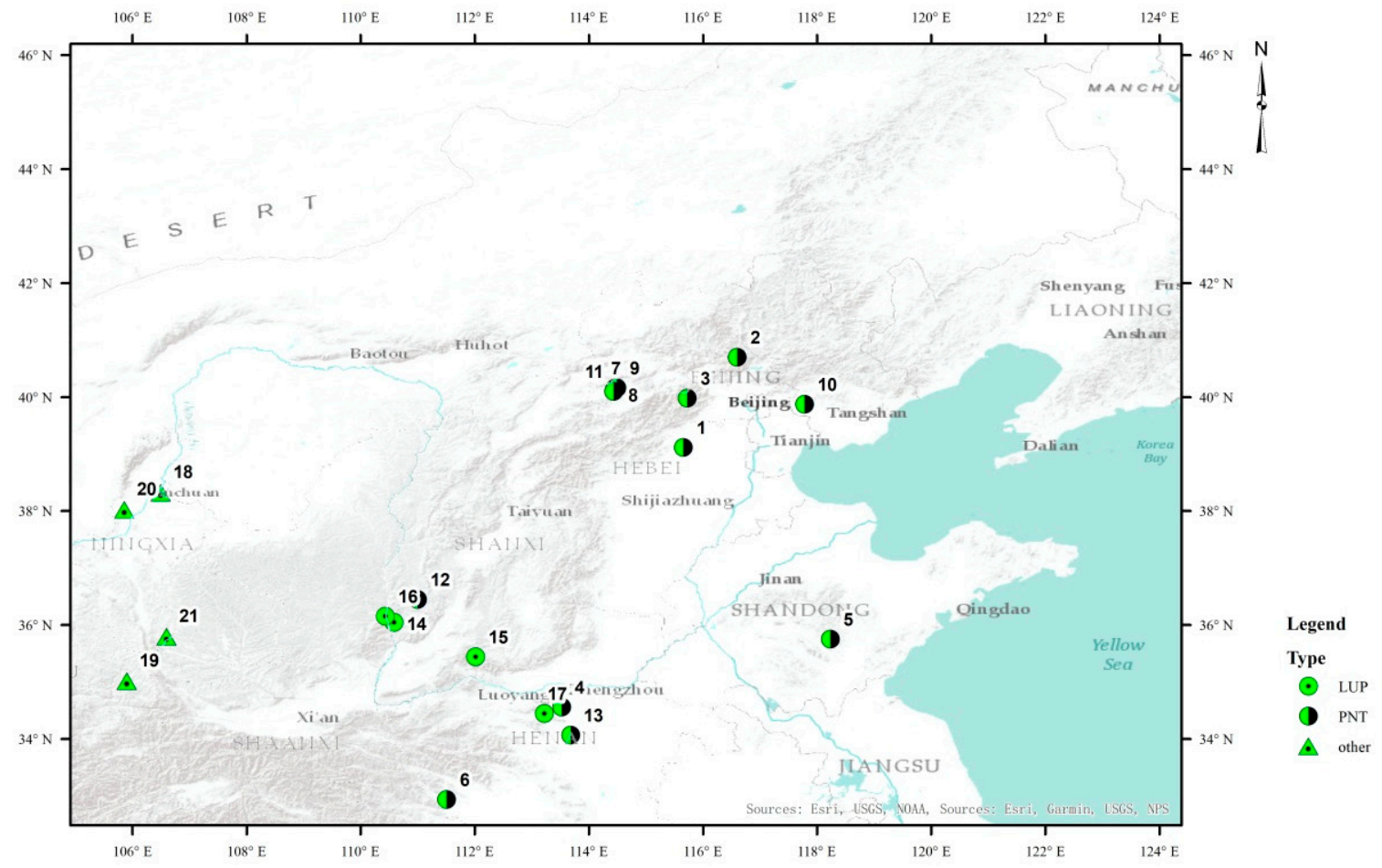

Figure 1. Archaeological sites dated to Pleistocene-Holocene transition in Northern China. PNT sites: 1 Nanzhuangtou; 2 Zhuannian; 3 Donghulin; 4 Lijiagou; 5 Bianbiandong; 6 Kengnan; 7 Yujiagou; 8 Ma'anshan; 9 Hutouliang; 10 Mengjiaquan; 11 Jijitan; 12 Xueguan; 13 Lingjing LUP sites: 14 Shizitan; 15 Xiachuan; 16 Longwangchan Other sites: 17 Xishi; 18 Shuidonggou Locality 12; 19 Dadiwan, Component 4; 20 Gezishan; 21 PY-04.

\section{Microblade-Based Societies during the Last Glacial Maximum}

Among archaeological sites in north China associated with microblade assemblages, the Shizitan site is best studied, especially in the terms of interdisciplinary approach focusing on human behaviors at a specific site. Shizitan is located at the Jixian County, SW Shanxi Province. Several localities are distributed along the Qingshuihe River, a tributary of the Yellow River. In recent years, Li Liu, in collaboration with local archaeologists, has been conducting a research project on plant use at this site, based on use-wear and residue analyses, suggesting that prehistoric plant use in northern China can be traced back to the immediately pre-LGM period, about $28 \mathrm{ky}$ cal. BP [31-34]. The plant use, including harvesting and processing using stone tools, can be divided into 6 phases (Table 4). This work suggests near-continuous exploitation of various plants in the Middle Yellow River Valley (both the Xiachuan site and the Longwangchan site share many material features with the Shizitan site, including microblades, grinding stones, and hematite processing) [31]. Their investigation provides substantial evidence of an early broad-spectrum subsistence strategy in the Upper Paleolithic northern China. 
Table 4. Phases of plant use at the Shizitan (SZT) site (data collected from [31]).

\begin{tabular}{|c|c|c|c|c|}
\hline Phase & Site and Stratum & Material & Function for Plants & Plant Use \\
\hline $\begin{array}{c}\text { Phase I } \\
\text { (pre-Last Glacial } \\
\text { Maximum (LGM)) }\end{array}$ & Stratum 8, SZT 29 & $\begin{array}{l}2 \mathrm{FL} \\
2 \mathrm{SL}\end{array}$ & $\begin{array}{l}\text { FL: cutting, scraping } \\
\text { SL: processing }\end{array}$ & $\begin{array}{l}\text { Starchy plants, including } \\
\text { Panicoideae (possibly Job's } \\
\text { tears), Triticeae and yam } \\
\text { No clear evidence for wild } \\
\text { millet collection }\end{array}$ \\
\hline $\begin{array}{l}\text { Phase II } \\
\text { (the early part } \\
\text { of LGM) }\end{array}$ & Stratum 7, SZT 29 & $\begin{array}{l}7 \mathrm{MB} \\
3 \mathrm{FL} \\
4 \mathrm{SL} \\
1 \mathrm{HS}\end{array}$ & $\begin{array}{l}\text { MB: cutting } \\
\text { FL: plant cutting } \\
\text { SL: processing * } \\
\text { HS: processing }\end{array}$ & $\begin{array}{c}\text { Wild cereals (e.g., Triticeae, } \\
\text { Job's tears and wild } \\
\text { millets), tubers and other } \\
\text { plants }\end{array}$ \\
\hline $\begin{array}{c}\text { Phase III } \\
\text { (the late part } \\
\text { of LGM) }\end{array}$ & $\begin{array}{l}\text { Strata } 6,5, \text { and } 4, \\
\text { SZT } 29\end{array}$ & $\begin{array}{l}1 \mathrm{MB} \\
7 \mathrm{FL} \\
3 \mathrm{SL}\end{array}$ & $\begin{array}{l}\text { MB: cutting } \\
\text { FL: cutting } \\
\text { SL: processing }\end{array}$ & $\begin{array}{l}\text { Wild cereals, and } \\
\text { especially Panicoideae }\end{array}$ \\
\hline $\begin{array}{c}\text { Phase IV } \\
\text { (the terminal LGM) }\end{array}$ & $\begin{array}{l}\text { Strata } 3 \text { and } 2, \\
\text { SZT } 29\end{array}$ & $\begin{array}{l}4 \mathrm{FL} \\
4 \mathrm{SL}\end{array}$ & $\begin{array}{l}\text { FL: cutting } \\
\text { SL: processing }\end{array}$ & $\begin{array}{c}\text { Grasses, including } \\
\text { Panicoideae and Triticeae. } \\
\text { Tubers are much less well } \\
\text { represented }\end{array}$ \\
\hline $\begin{array}{l}\text { Phase V } \\
\text { (warmer } \\
\text { conditions) }\end{array}$ & Stratum 1, SZT 29 & $2 \mathrm{FL}$ & FL: cutting & $\begin{array}{l}\text { Unknown, only one } \\
\text { non-specific phytolith was } \\
\text { recovered. }\end{array}$ \\
\hline $\begin{array}{c}\text { Phase VI } \\
\text { (Early Holocene) }\end{array}$ & Stratum 1, SZT 5 & $2 \mathrm{FL}$ & FL: cutting & Unknown, only starch \\
\hline
\end{tabular}

Notes: FL: flake, MB: microblade, SL: slab or slab fragment, HS: handstone. * Both slab fragments and handstones are used for processing plant food and minerals.

Liu et al. [33] propose the viewpoint, "The trajectory from intensified collection of a wide range of wild plants to domestication of a small number of species was a very long process in north China", and suggest that it "parallels the transition from the 'broad spectrum revolution' to agriculture in the Near East" (pp. 3524). Although they did not use the term "intensification" to generalize this process, plant processing could be seen as a means of food resource intensification, since people need to invest much labor to extract energy from plants, including tubers and seeds. Plant use might suggest a local population packing effect during the LGM in the Middle Yellow River Valley, possibly caused by southward movement of prehistoric peoples from the north or the west, which can be tested as a hypothesis.

The effective temperature (ET) value of the Shizitan site under LGM climatic conditions was below the terrestrial plant threshold, suggesting that the prehistoric foraging societies needed to depend on terrestrial animal resources during the LGM. However, a relatively longer growing season than that of Siberia provides this location higher net above-ground productivity and primary biomass. The Shizitan site is also located in the belt of high secondary biomass, making it a place that possessed plenty of both terrestrial plants and animals. The Qingshuihe River, a small tributary of the Yellow River, also provided people with enough water and aquatic resources for survival, although archaeologists have not found substantial evidence of fishing. The maps in Figures 2-6 support the following characterization of the environment and resource use at the Shizitan site:

"Around 23,000-18,000 years ago during the LGM, the Qingshui River valley appears to have been an area with a wide range of faunal and floral resources, which attracted small hunting-gathering groups. In addition to hunting, people collected and processed many types of plants, including grass seeds of Triticeae and Paniceae, Vigna beans, D. opposita yam, and T. kirilowii snakegourd roots, among others". [32] (p. 5384) 

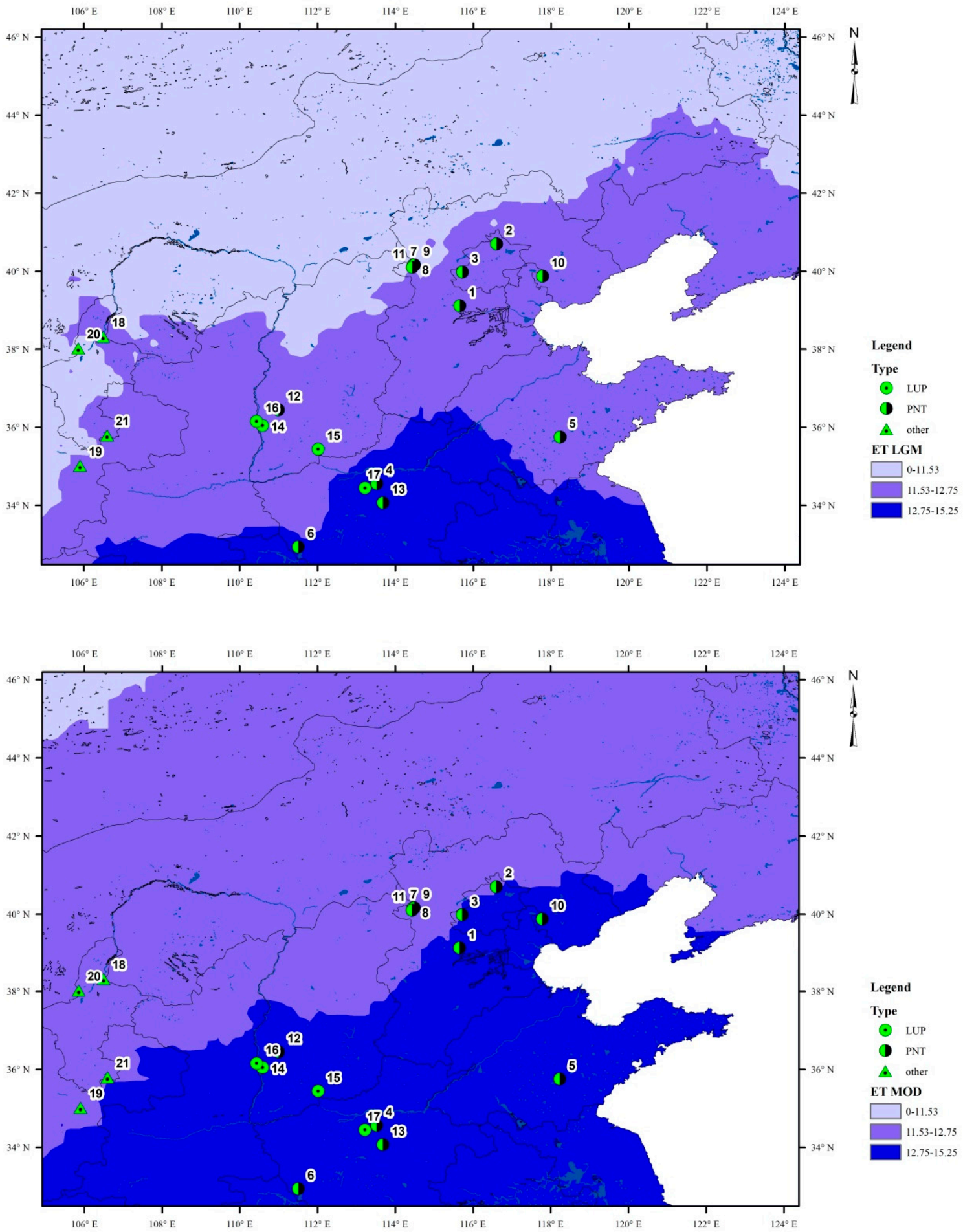

Figure 2. Archaeological sites dated to the Pleistocene to Holocene transition on the maps of effective temperature (ET) in Northern China under LGM and modern climatic conditions. 

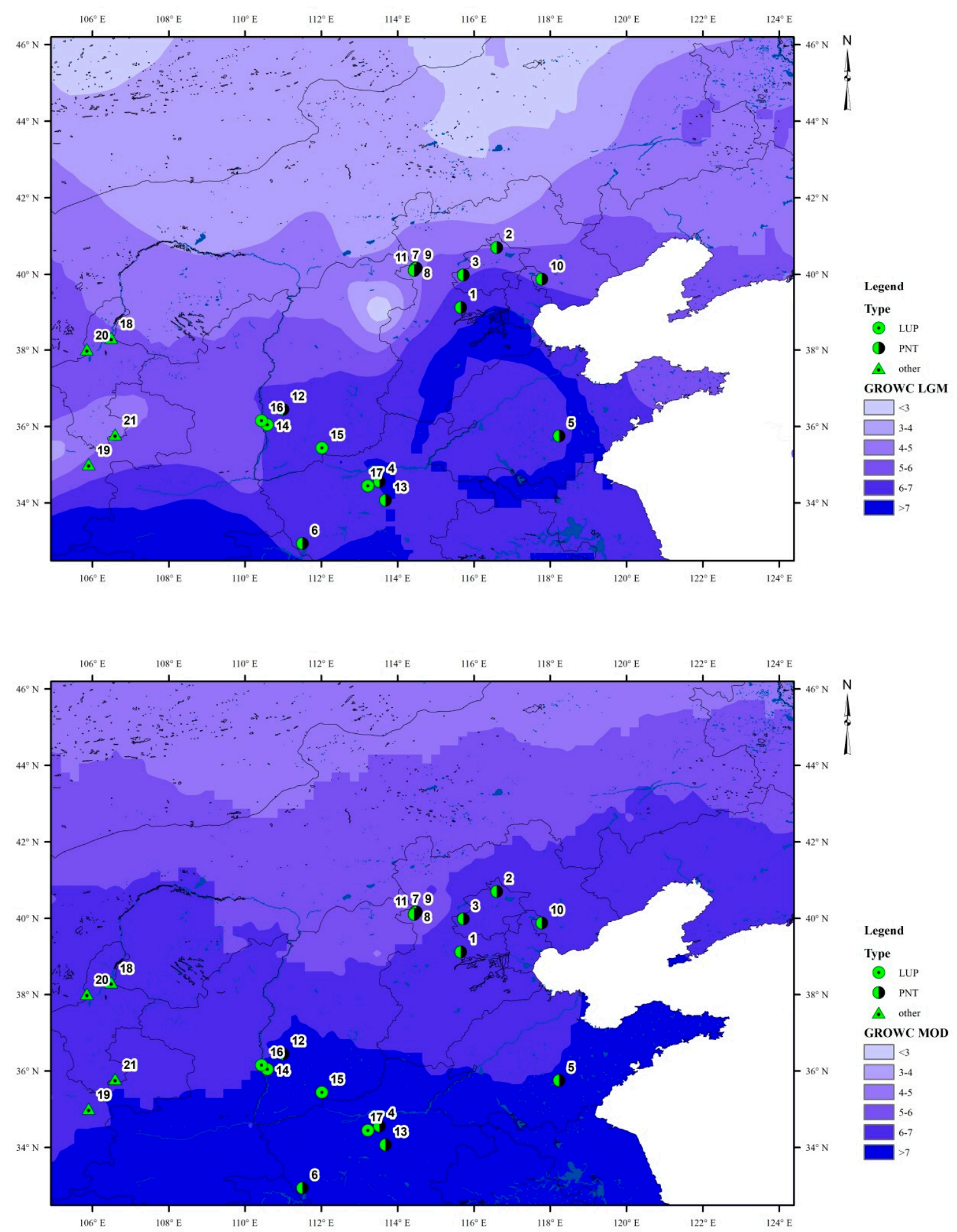

Figure 3. Archaeological sites dated to the Pleistocene to Holocene transition on the maps of growing season (GROWC) of Northern China under LGM and modern climatic conditions. 

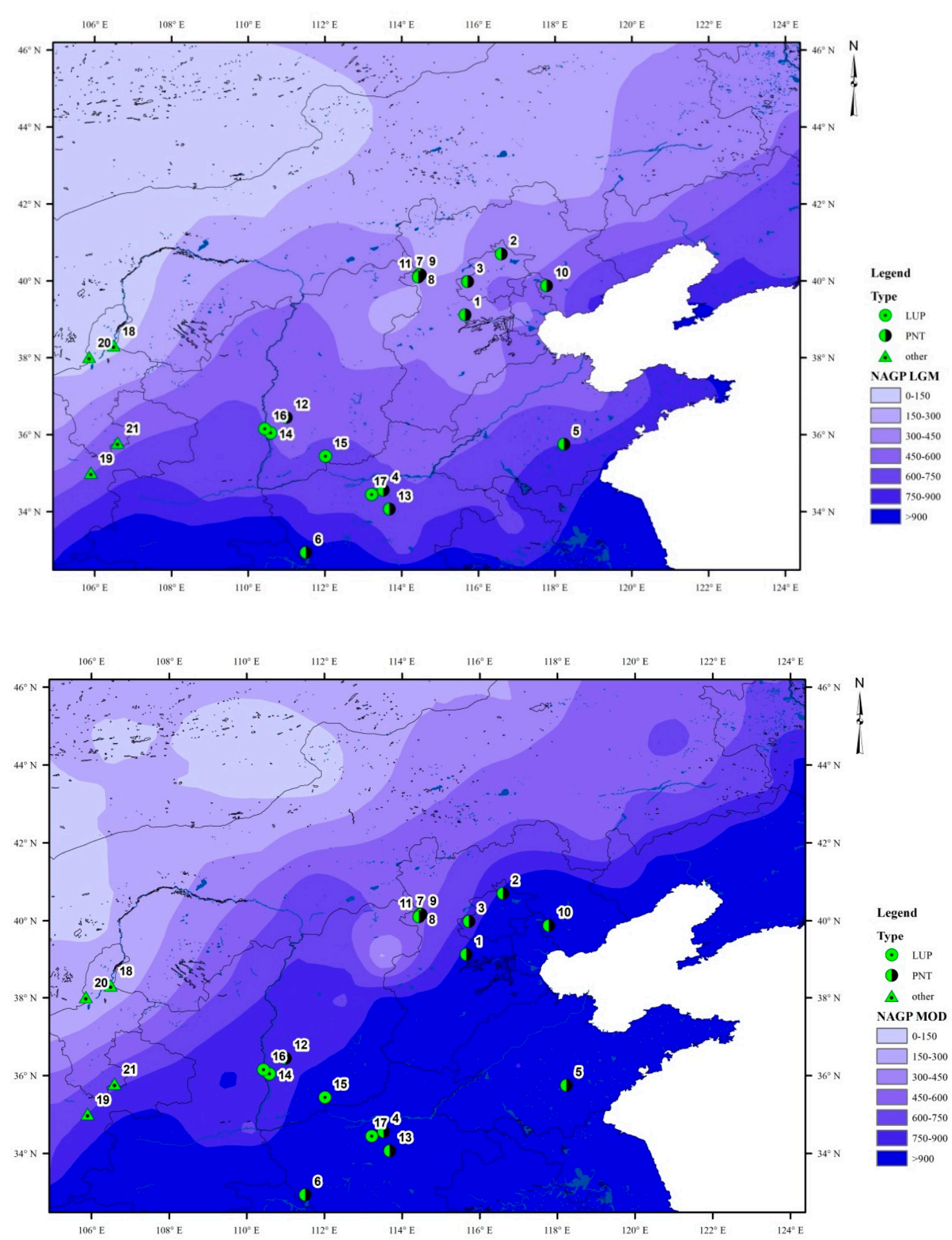

Figure 4. Archaeological sites dated to the Pleistocene to Holocene transition on the maps of net above-ground productivity (NAGP) in Northern China under LGM and modern climatic conditions. 

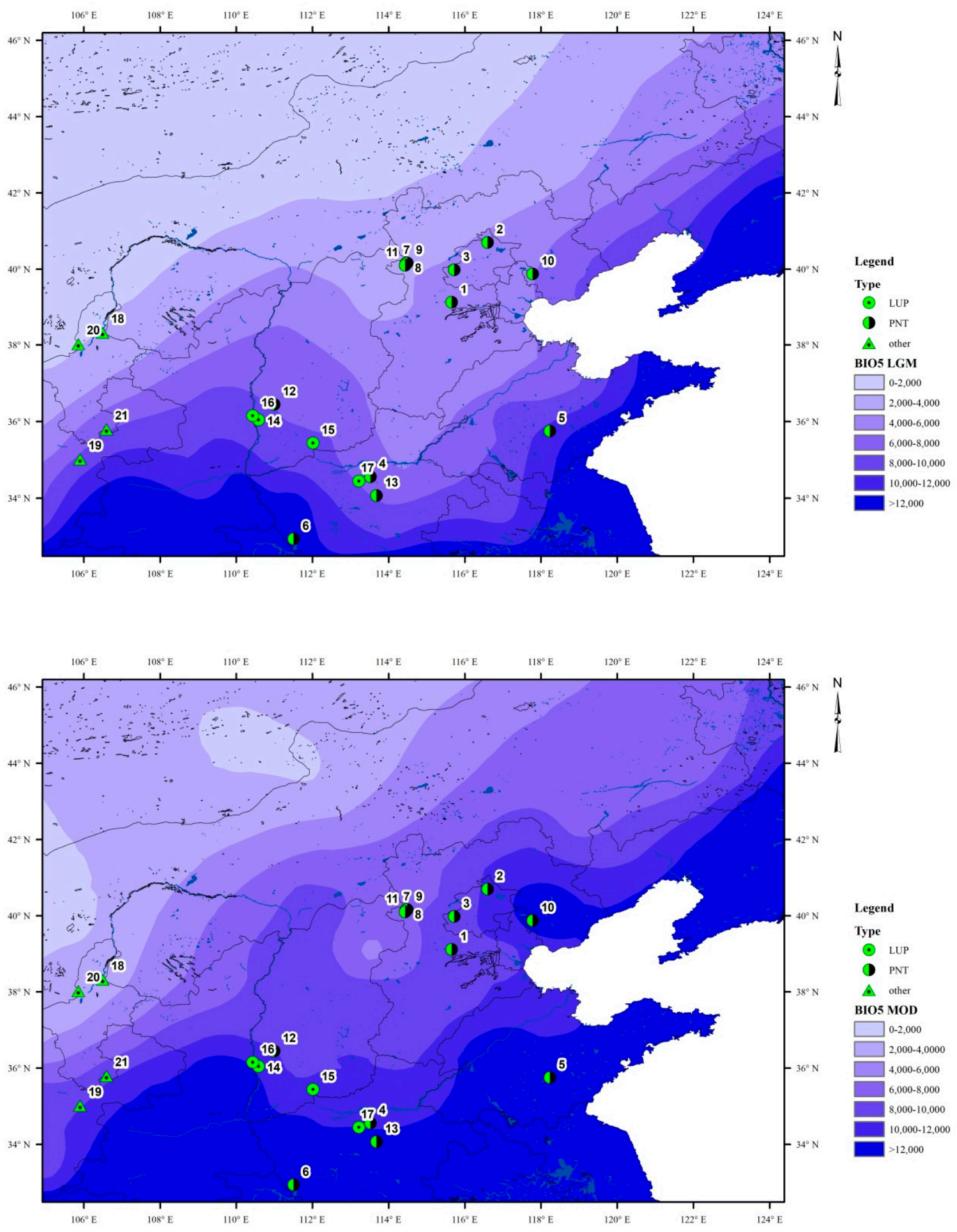

Figure 5. Archaeological sites dated to the Pleistocene to Holocene transition on the maps of primary biomass (BIO5) of Northern China under LGM and modern climatic conditions.

Thus, there is no question that prehistoric hunter-gatherers in this region adopted a mixed economy to satisfy their subsistence requirements, which might have been a general strategy adopted by the peoples during the LGM. The peoples in the lower latitudes or above the subpolar bottleneck $\left(\mathrm{ET}=11.53^{\circ} \mathrm{C}\right)$ have significantly more terrestrial plant resources to utilize. 

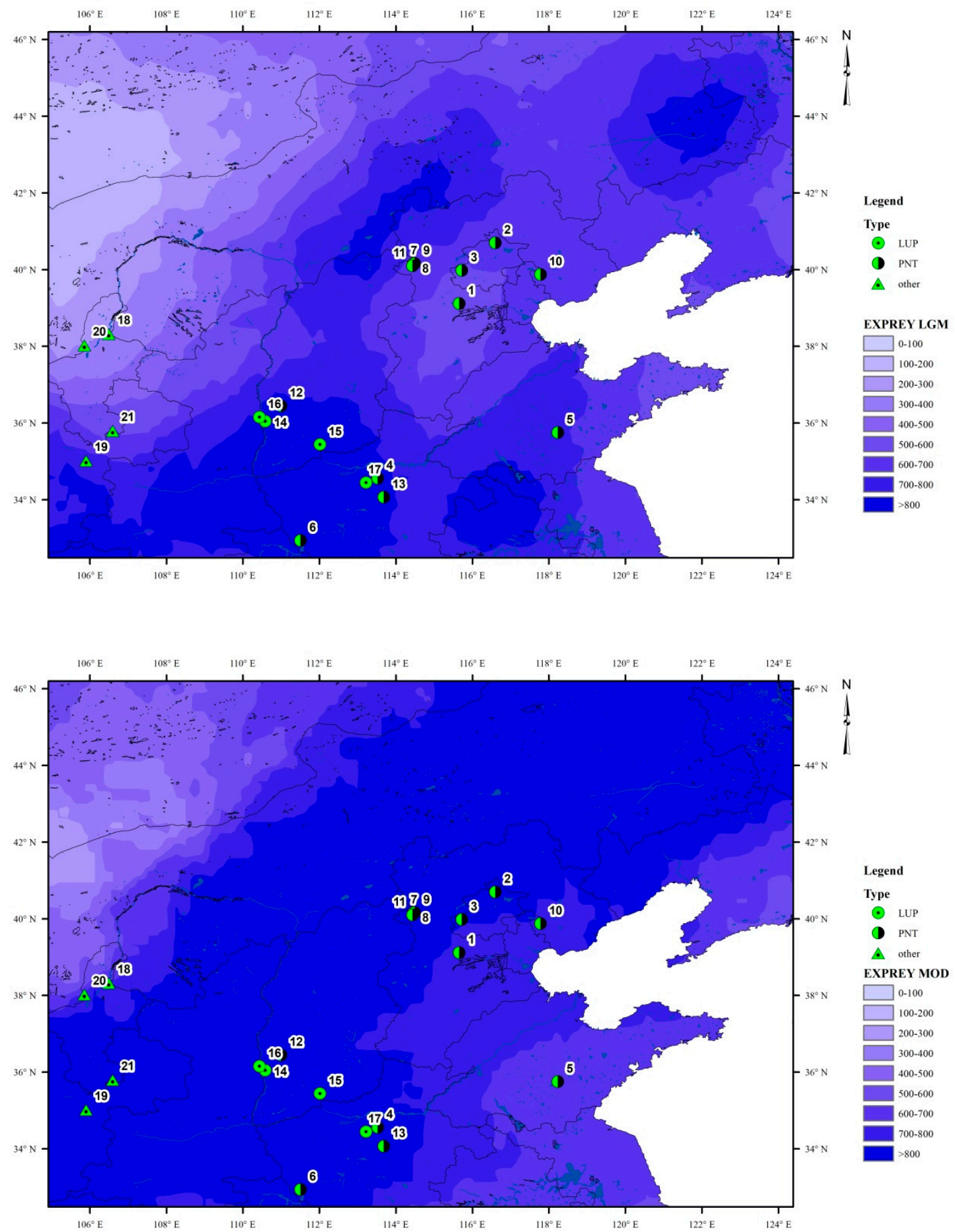

Figure 6. Archaeological sites dated to the Pleistocene to Holocene transition on the maps of secondary biomass (expected moderate body-size ungulate biomass, EXPREY) of northern China under LGM and modern climatic conditions.

The question is whether resource intensification on terrestrial plants was practiced during the LGM. According to the maps produced under the growth rate model, unpacked/packed maps show that the location of the Shizitan site is expected to have favored a gathering-dominated economy if population became packed, while it would have favored a hunting-dominated economy if population was unpacked (Figure 7). The ethnographic projected population density map under LGM climatic conditions (Figure 8) suggests that local resource cannot support a high population density in the region where the Shizitan site is located $\left(2-3.033\right.$ persons per $\left.100 \mathrm{~km}^{2}\right)$. The patterns resulting from 
these projections can only be used if hunter-gatherers in this location were organized like recent hunter-gatherers in similar contexts, rather than used to say what the archaeological record actually was. In addition, the packing threshold was developed using data for mobile plant-dependent hunter-gatherers who used no long-term storage-the density threshold at which highly mobile hunters might need to intensify would be lower. In other words, the occupants at the Shizitan site during the LGM might have intensified plant resources especially for storage. But more archaeological evidence is needed to support this hypothesis.
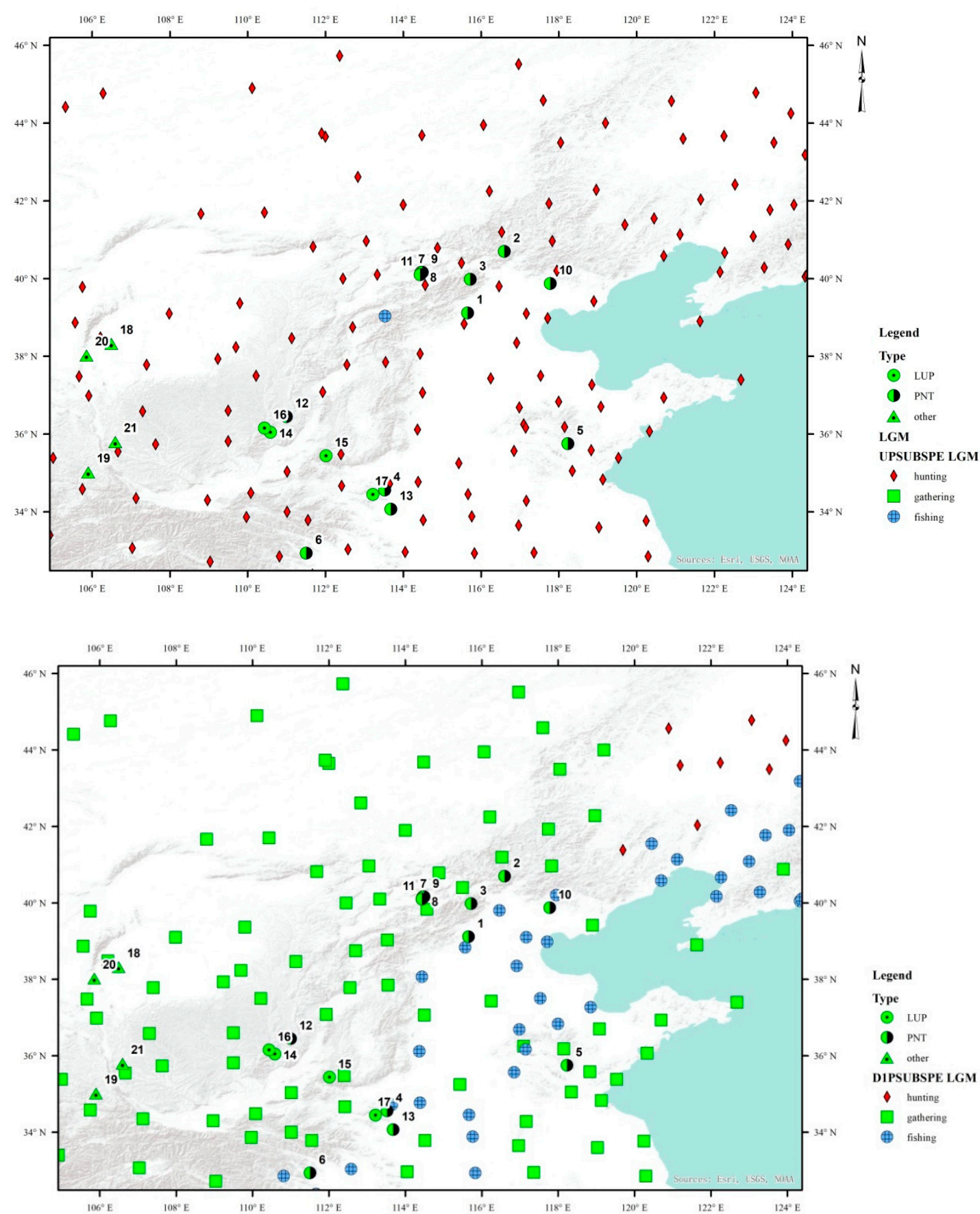

Figure 7. Archaeological sites dated to the Pleistocene to Holocene transition on the maps of projected unpacked and packed HG subsistence specialty (UPSUBSPE and D1PSUBSPE) in northern China under LGM climatic conditions. 

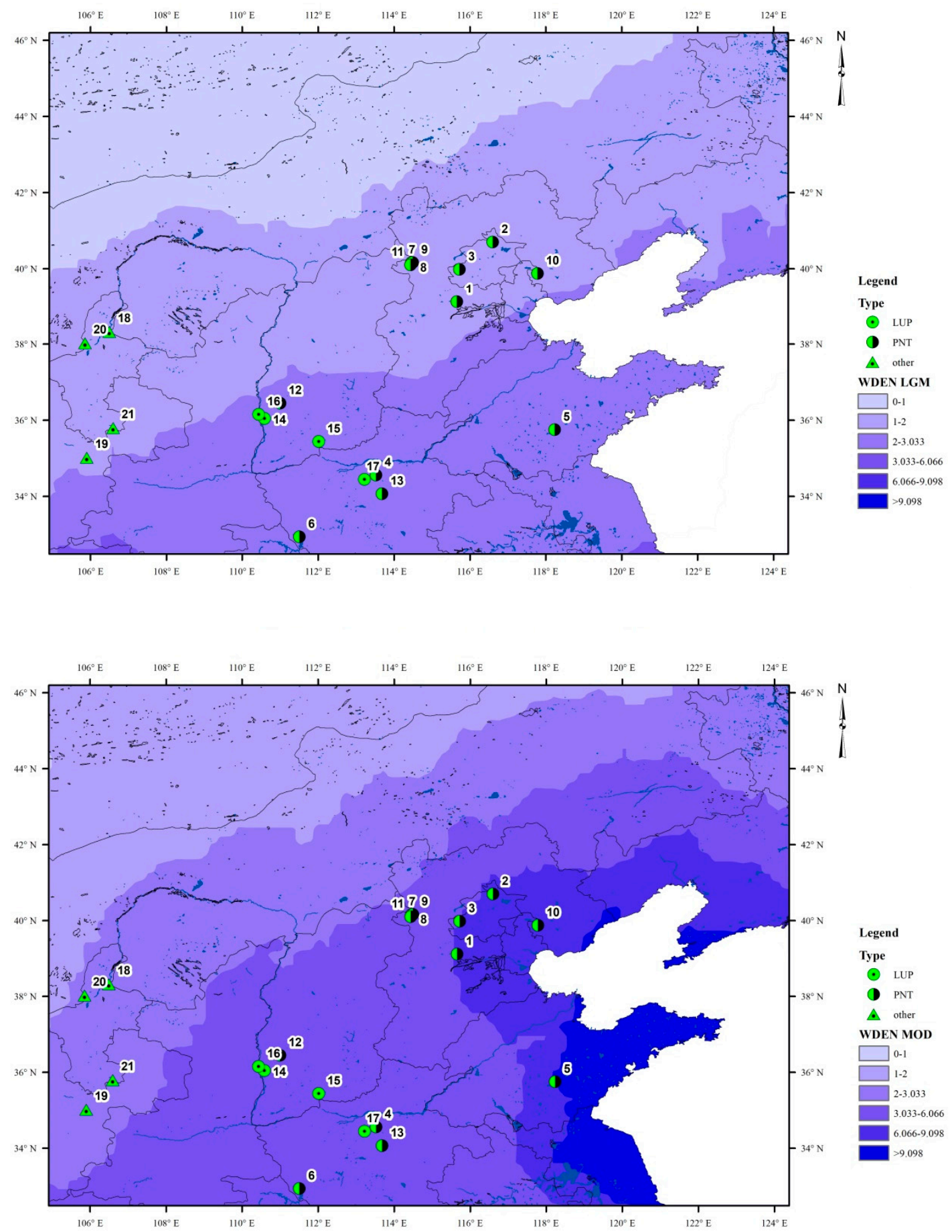

Figure 8. Archaeological sites dated to the Pleistocene to Holocene transition on the maps of projected HG population density (WDEN) of northern China under LGM and modern climatic conditions.

Is there any other possible explanation for the existence of intensive plant use at the Shizitan site? The map of EXDMOV1 (projected total distance moved, scaled for subsistence type, for groups with year round camp to camp mobility pattern) shows that foraging societies in northern China during the LGM had to adopt highly mobile lifeways (comparing with the values of EXDMOV1 under current climatic conditions) (Figure 9). Widespread microblade technology provided them with effective and light composite weaponry to hunt animals; some microblades were used as reaping knife inserts to harvest and process plant resources. Microblade-based societies successfully adapted to the deteriorated environment with relatively lower primary and secondary biomass. The region 
surrounding the Shizitan has a relatively lower value in terms of expected distance of residential movement (250-300 miles) than its neighboring regions to the east and northwest, which suggests that the occupational duration in one location might be longer and occasional sedentism might have happened during the LGM (Figure 9). Maps of population group size in the subsistence-settlement system (Figure 10) suggest that under LGM climatic conditions, prehistoric foraging societies near the Shizitan site, as well as the Longwangchan and Xiachuan sites, harbored 20-25 persons in the most dispersed settlement phase, $90-120$ persons in the most aggregated phase, both less than its eastern neighboring regions. However, the site is located in the zone with high GROUP3 values (400-500 persons), representing a high probability of annual or every several year's aggregation. Although it is located at the edge of GROUP3 isoline (400 persons), the Qingshuihe-Yellow River system and valley environment offer an advantage with regard to supporting populations, especially aggregations. The Shizitan site and Longwangchan site Locality 1 share almost the same archaeological record, suggesting that prehistoric foragers could easily have crossed the Yellow River in winter on ice under very low temperatures during the LGM and post-LGM Pleistocene (personal communication with Y.-H. Song). The combination of high GROUP3 size in this region and its river valley topography might have made the Shizitan site an ideal place for multi-group aggregations (annually or every few years). This hypothesis is consistent with the archaeological record associated with 285 hearths at Locality S29 (Table 5). Microblade assemblages imply the existence of organized hunting activities, and plant remains studied in Li Liu's project might prove the association between plant-use behaviors and the activities surrounding the hearths in localities S14 and S29 in further research [31-33]. At least, this hypothesis has been partly supported by the recent analysis of Locality S29 [25], but detailed discussion of human aggregation at the Shizitan site is needed.

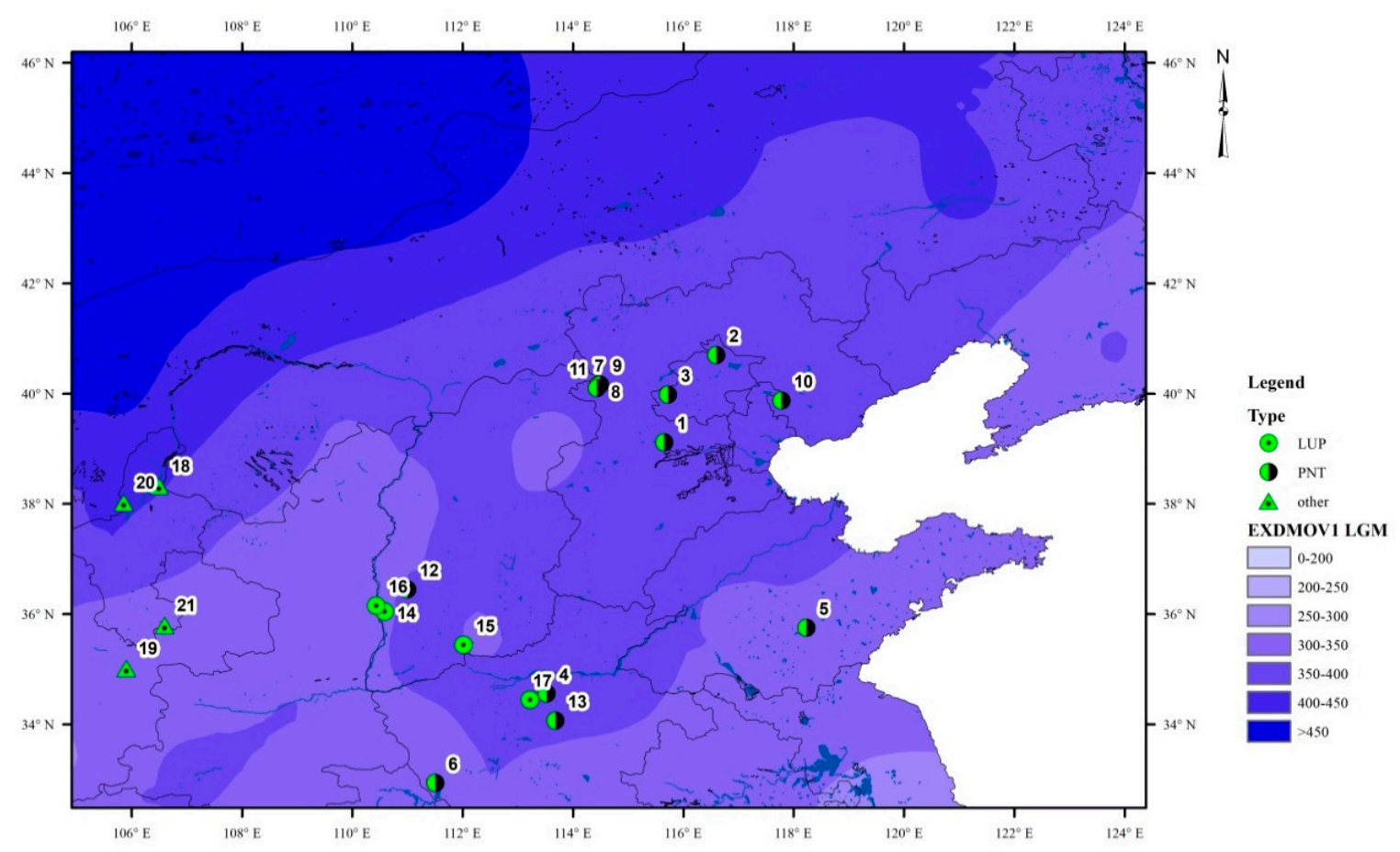

Figure 9. Cont. 


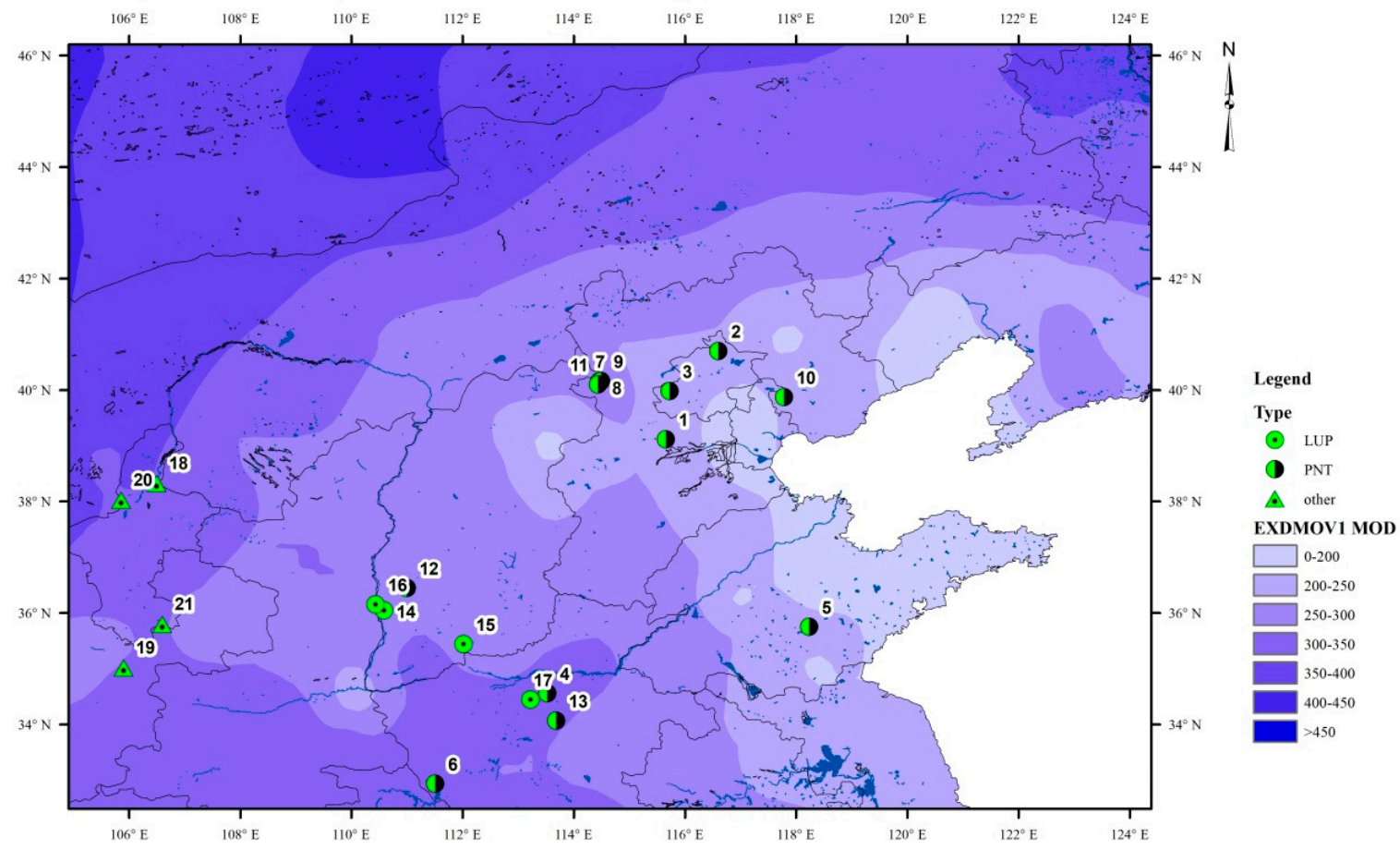

Figure 9. Archaeological sites dated to the Pleistocene to Holocene transition on the maps of expected distance of residential movement per year of hunter-gatherers (EXDMOV1) in northern China under LGM and modern climatic conditions.

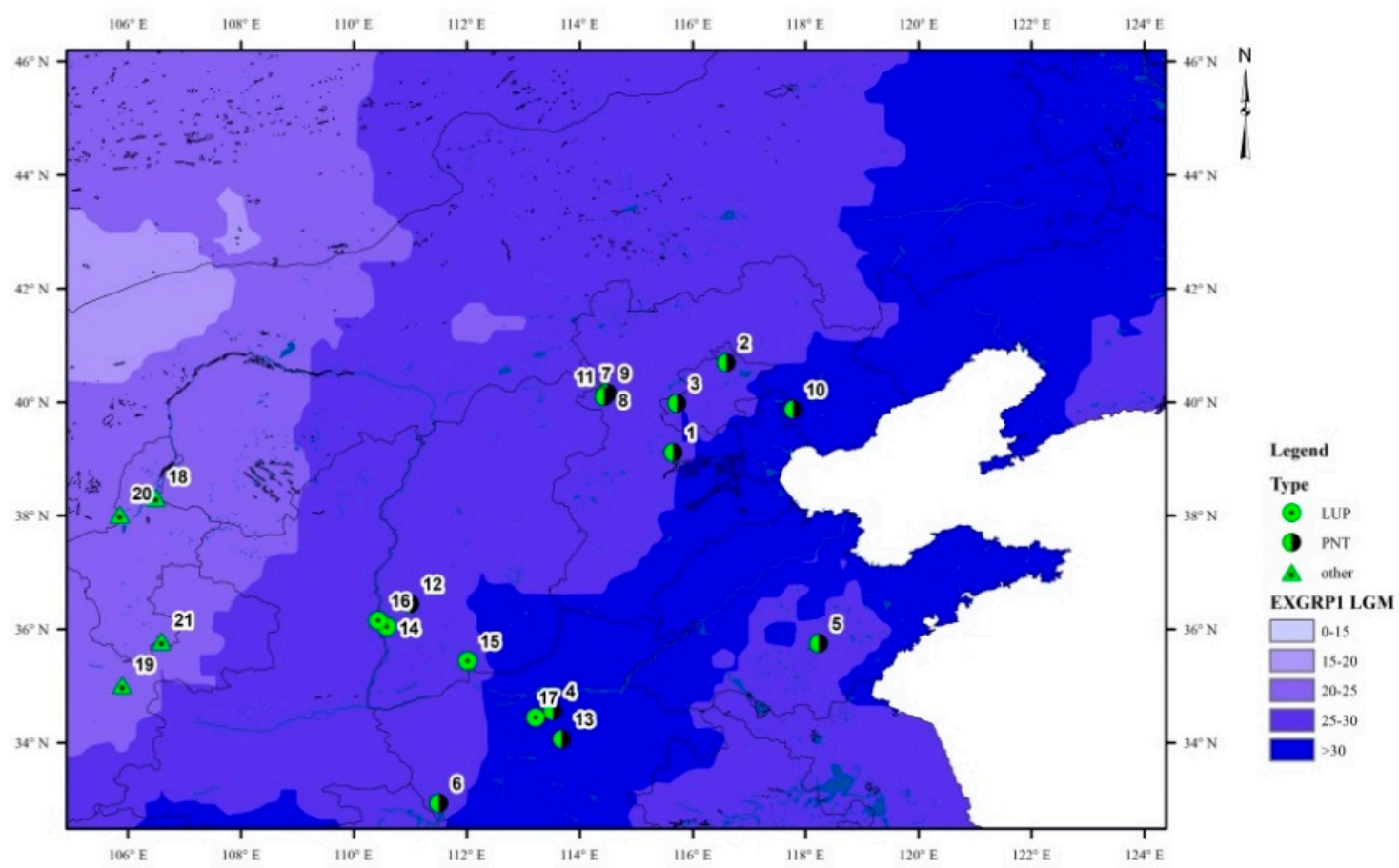

Figure 10. Cont. 

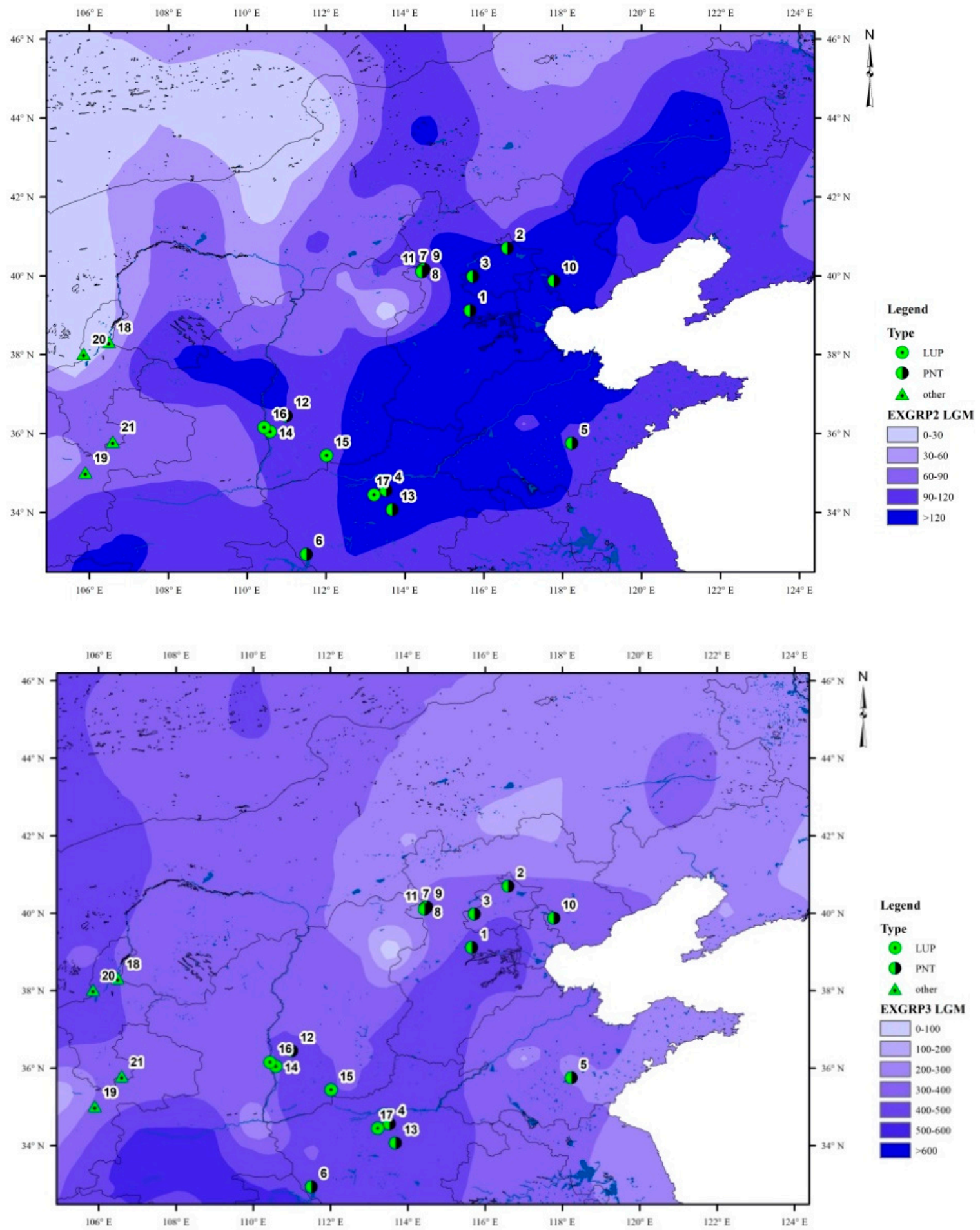

Figure 10. Archaeological sites dated to the Pleistocene to Holocene transition on the maps of expected hunter-gatherer group size in northern China under LGM climatic conditions. 
Table 5. Hearths in the Shizitan site, Locality S29 [25].

\begin{tabular}{ccc}
\hline Type of Hearth & Number & Percentage \\
\hline Above-ground hearth & 265 & $92.98 \%$ \\
Above-ground hearth with sandstone rocks & 13 & $4.56 \%$ \\
Round-pit hearth & 5 & $1.76 \%$ \\
Hearth with stone-floored pit & 2 & $0.7 \%$ \\
\hline Total & 285 & $100 \%$ \\
\hline
\end{tabular}

To sum up, the macroecological approach suggests that intensification might not have existed at the Shizitan site during the LGM. The archaeological record of plant resource exploitation at the Shizitan site indicates that increasing population densities might have made intensification important. However, plants may have been used as supplementary food resources (tubers and wild millet) in daily life. Just as the maps suggested, the plant foods also might have been used to satisfy the regional band requirements of periodic aggregations at the Shizitan site (e.g., Localities S29 and Loc. 14). Unfortunately, because of a lack of lithic refitting research, the hypothesis proposed here remains speculative, although the limited available evidence is suggestive. Limited by the preliminarily published data, testing this hypothesis will require more evidence and question-oriented studies in the future. Plant use at the Shizitan site lasted until the early Holocene, when it was totally abandoned. This is a key site for archaeologists to investigate the process of a potential broad spectrum revolution in the Upper Paleolithic of the Middle Yellow River Basin, and/or to study subsistence-settlement systems and technological organization of microblade-based societies in northern China, since it possesses a complete record from Phase I to Phase III (and some in Phase IV).

\section{Broad Spectrum Revolution and Intensification during the Pleistocene-Holocene Transition}

The Late Pleistocene and Early Holocene in northern China witnessed the transformation from societies composed of mobile hunter-gatherers equipped with microblade technology to societies equipped with ground stone tools, pottery, food production, and durable dwellings. There have been many studies on these aspects of the transition. Although their appearances can be dated to different specific times in different regions, the formation of agricultural societies can be seen as an emergent event, rather than as the simple overlap of historical events. Chen and $\mathrm{Yu}[1,2]$ have termed this period the Paleolithic to Neolithic transition (PNT). They "synthesize a broad range of diagnostic artifacts, settlements, site structure, and biological remains to develop a working hypothesis that agriculture was differentially developed or adopted according to 'initial conditions' of habitat, resource structure, and cultural organization" [2]. The authors noted multiple and divergent evolutionary pathways, including northern China (divided at the Taihangshan Mts. into the North China Plain and Loess Plateau). Published data on the chronology and archaeological record of northern China linked to the PNT are presented, clearly showing basic information on diagnostic artifacts, settlements, and site structure associated with social organization, and potential species for domestication Tables 1 and 2 in ref [2], with updated information from recently published results. The two papers by Chen and Yu [1,2] clearly and successfully illustrate cultural change during the PNT of Northern and Southern China, but the authors did not fully apply a macroecological approach in their study.

Since I have shown a series of maps of Northern China for the Shizitan case under LGM conditions, I now only show the corresponding maps under modern climatic conditions, representing the early Holocene. Readers can compare the maps under the two sets of climatic conditions.

The Pleistocene-Holocene transition witnessed a dramatic change from glacial to interglacial, especially after the Younger Dryas. The Terminal Pleistocene after the LGM (starting at $15 \mathrm{ky}$ uncalibrated BP), experienced several climatic phases of relatively warm and cold periods. The Younger Dryas is seen as a stimulus for food production in Northern China and the Near East [7]. In this project, the maps of LGM climatic conditions can be read similarly to those under Younger Dryas conditions, since paleoclimate data suggests that the Younger Dryas was associated with marine isotopic values 
as low as those of the LGM, but during a much shorter time (about 1300 years, 12.9-11.6 ky cal. BP, or about 600 years, 10.8-10.2 ky uncalibrated BP) [34,35].

Effective temperature dramatically increased and the isoline of the terrestrial plant threshold $\left(\mathrm{ET}=12.75^{\circ} \mathrm{C}\right)$ moved northward. Several sites with the best evidence of food production are located around this isoline (Nanzhuangtou, Zhuannian, and Donghulin) (Figure 2). In contrast to the more localized and fragmentary distribution of growing season isolines under LGM climatic conditions, the length of the growing season dramatically increased, causing more gradual change along latitudes $\left(34^{\circ} \mathrm{N}-40^{\circ} \mathrm{N}\right)$ under interglacial conditions (Figure 3), which could help in the formation of belts favorable for stable plant growth and provide a good initial condition for cultivation. The increases of ET and length of growing season resulted in an increase of net above-ground productivity and primary biomass (Figures 4 and 5). Secondary biomass also increased and the central region moved northwest to the modern forest-steppe ecotone along the southwest-northeast China line Figures 5 and 7 in ref [6]. Under modern climatic conditions (early Holocene), the three sites-Nanzhuangtou, Zhuannian, and Donghulin - are all located at the edge of the zones with high values of both primary and secondary biomass, i.e., the transition zone (Figure 6). This phenomenon matches the basic hypothesis advanced by Binford [36,37], that early food production would appear in zones adjacent to areas with the most resources, as a cultural adaptation after mobility declined or ceased to be effective to solve the problems of population growth.

The growth rate model also shows the replacement of terrestrial hunting by gathering and aquatic resource use. Different from the maps under LGM climatic conditions, the subsistence replacement indeed happened during the Pleistocene to Holocene transition-Chen and Yu's Paleolithic to Neolithic Transition. For northern China (above $30^{\circ} \mathrm{N}$ ), hunting in some places was replaced by the gathering of terrestrial plants (e.g., the Gezishan site in Upper Yellow River Basin), by aquatic resource use (some sites in NE China, such as the Houtaomuga and Shuangta sites), or food production (i.e., Nanzhuangtou, Zhuannian, Donghulin, and other PNT sites, as well as the early Neolithic sites) (Figure 11). The hunters north to the Yanshan Mts. (north to the area of Beijing) retained their hunting-dominated lifeways in a mixed economy with the aid of advanced microblade technology (pencil-shaped microcores) during the early and middle Holocene, perhaps as specialized hunters who exchanged wild meat with neighboring agriculturists, who were in turn finally replaced by pastoralism (about 1000 BC) [38]. The sites of Nanzhuangtou, Zhuannian, and Donghulin are all located in the boundary regions of combined hunting, gathering and fishing under the packed population condition, suggesting that they needed to manage a pluralistic subsistence style. This is supported by the coexistence of microblade-equipped hunting weaponry, pottery, mortars and pestles, and polished tools at the Zhuannian and Donghulin sites. The lack of any microblade assemblage at the Nanzhuangtou site perhaps resulted from the sampling representation of the excavated area (300 out of $2000 \mathrm{~m}^{2}$ ), or a higher level of food production with a very low level of hunting activities. In addition, evidence of hunting activity calls for further research focus, since some sites are located near water systems and people are expected to have exploited some aquatic resources.

The increase of sedentary life can also be investigated from the viewpoint of a macroecological approach, in terms of projected population density and expected distance of residential movement per year. The Nanzhuangtou, Zhuannian, and Donghulin sites are located at the position of 2/3 packing threshold, and the Bianbiandong cave site is at the packing threshold (Figure 8). Thus, the people at Bianbiandong should have experienced population pressure, while the occupants at sites Nanzhuangtou, Zhuannian, and Donghulin might have experienced population packing if they emphasized hunting during the early Holocene. If they were mobile hunter-gatherers, their mobility should have dramatically decreased from 250/300-350 miles travelled per year during the Younger Dryas (see the EXDMOV1 under LGM climatic conditions), to 200-250 miles per year during the early Holocene (Figure 9). However, if they were sedentary hunter-gatherers their mobility only needed to be $20-40$ miles per year during early Holocene (Figure 12). For people who practiced cultivation, the decrease in mobility could focus on a habitat where they could use low-energy food resources 
through dietary expansion and/or intensification. Therefore, zones with relatively lower primary and secondary biomass compared to neighboring regions associated with optimal resource distribution could serve as new locations in which 'daughter' groups could practice food production [37,38]. The lifeways built on sedentism (decreased mobility) favored durable ground/polished and expedient stone tools, rather than highly curated and formal chipped stone tools designed for mobile hunting, because the most important part of the economy became plant resource-use and even cultivation $[39,40]$. This may explain the disappearance of microblade assemblages in early Neolithic sites in northern China.
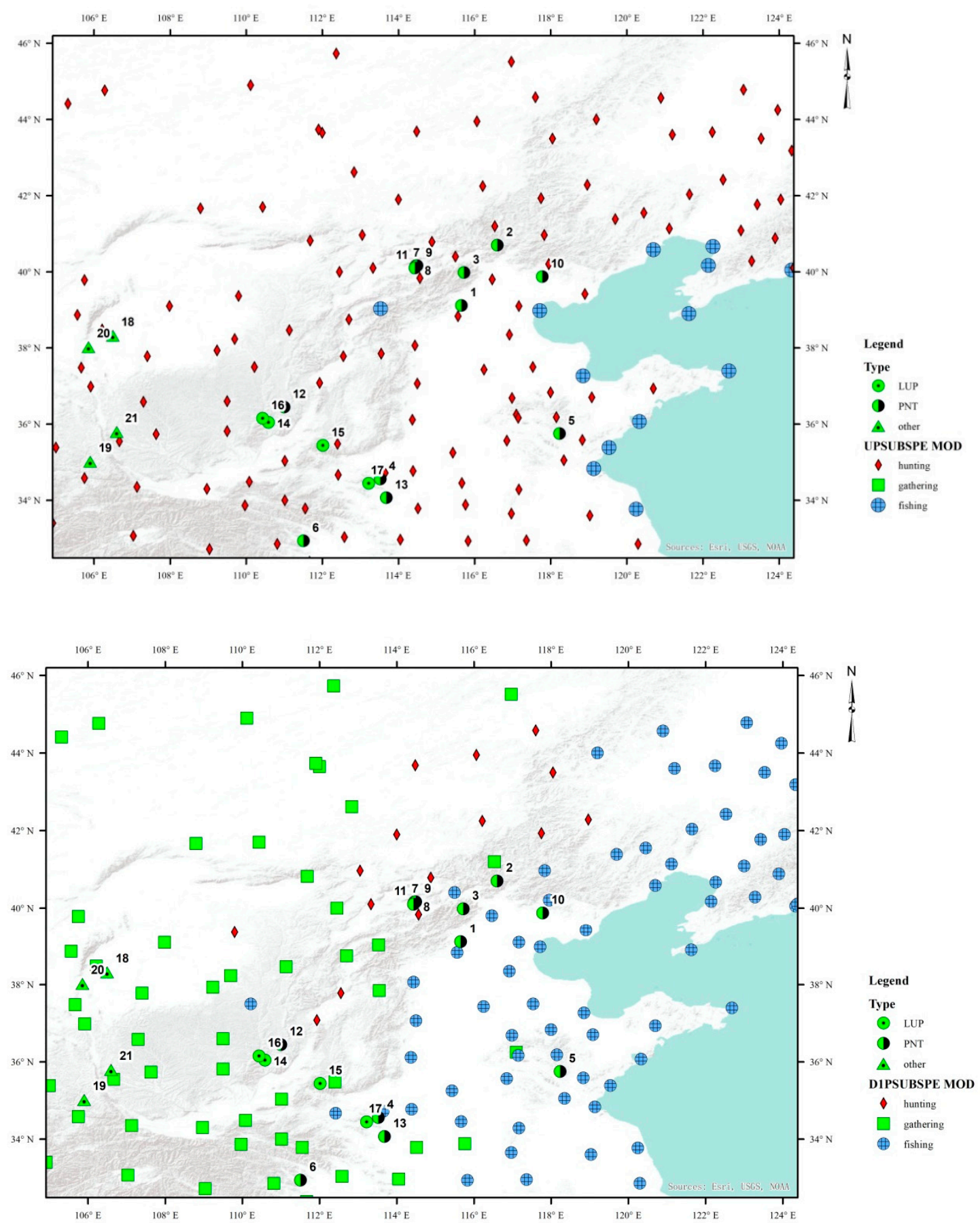

Figure 11. Archaeological sites dated to the Pleistocene to Holocene transition on the maps of projected unpacked and packed hunter-gatherer subsistence specialty (UPSUBSPE and D1PSUBSPE) in northern China under modern climatic conditions. 


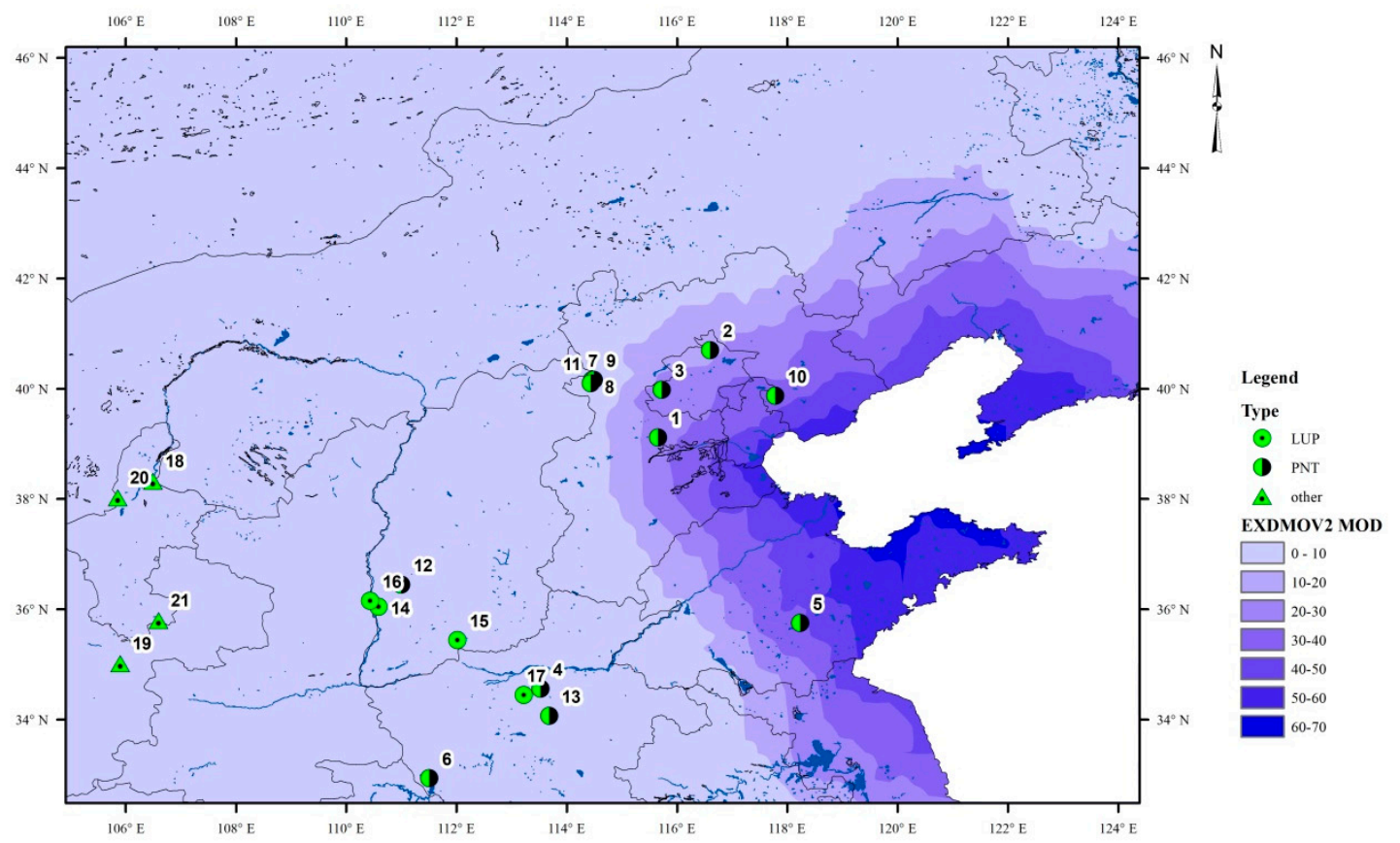

Figure 12. Archaeological sites dated to the Pleistocene-Holocene transition on the map of EXDMOV2 (expected distance of residential movement per year of hunter-gatherers with central location or who are primarily sedentary) in northern China under modern climatic conditions.

\section{The Demise of Microblade-Based Societies}

The Early Holocene witnessed the disappearance of microblade technologies in North China and the demise of microblade-based societies, although there were a few later sites associated with some of these technologies. However, investigating this cultural process is tough, since those specializing in Neolithic archaeology rarely report those data in the early stages. There are few published data on microblades or microcores in the Neolithic sites (8500-2000 BP) of the northern China cultural region. Although this can be explained as a reporting bias of Neolithic archaeologists, in fact there may be a real absence or very few microblades in the sites. In contrast, Holocene microblade assemblages have been reported in some sites of the Inner Mongolia Steppe, in NE and NW China, on the Tibetan Plateau, and in SW China, all of which are frontier regions of China. Details of the disappearance of microblade technology are little known, let alone the process of the decline and disappearance of microblade-based societies. Among the sites dated to the late PNT after the Younger Dryas event, only the Donghulin site has been researched in detail in Tian-Xing Cui's [15] dissertation. Microblade assemblages at this site co-exist with ground stone and other percussion tools. By contrast with the classic microblade technology at sites dated to terminal Pleistocene, such as Hutouliang [41], Lingjing [42], Yujiagou [20], and Shuidonggou Locality $12[28,43]$, the microcores at the Donghulin site show great variety with unstandardized production methods (Cui used many subtypes to classify them), and some tools show expedient characteristics. The degeneration of microblade production methods might be a result of the transition to a patchy environment composed of forest and grassland. Forest animals dominate the fauna of the Donghulin site (Figure 13), which might impede the use of weaponry equipped with microblades, since open landscape was being replaced by forest and marshes that favor alternative hunting technologies. Paleoethnobotanical research suggests that the occupants at the Donghulin site intensified the use of plant products. Macrobotanical remains including Gramineae, Leguminosae, and Celtis sinensis, and microbotanical remains (starch) on mortars and pestles including Triticeae and wild foxtail millet (Setaria italica), imply a broad-spectrum economy and intensification. Sedentism had already been adopted by the late PNT occupants, which also reduced the use of microblade technology designed for highly mobile lifeways. 

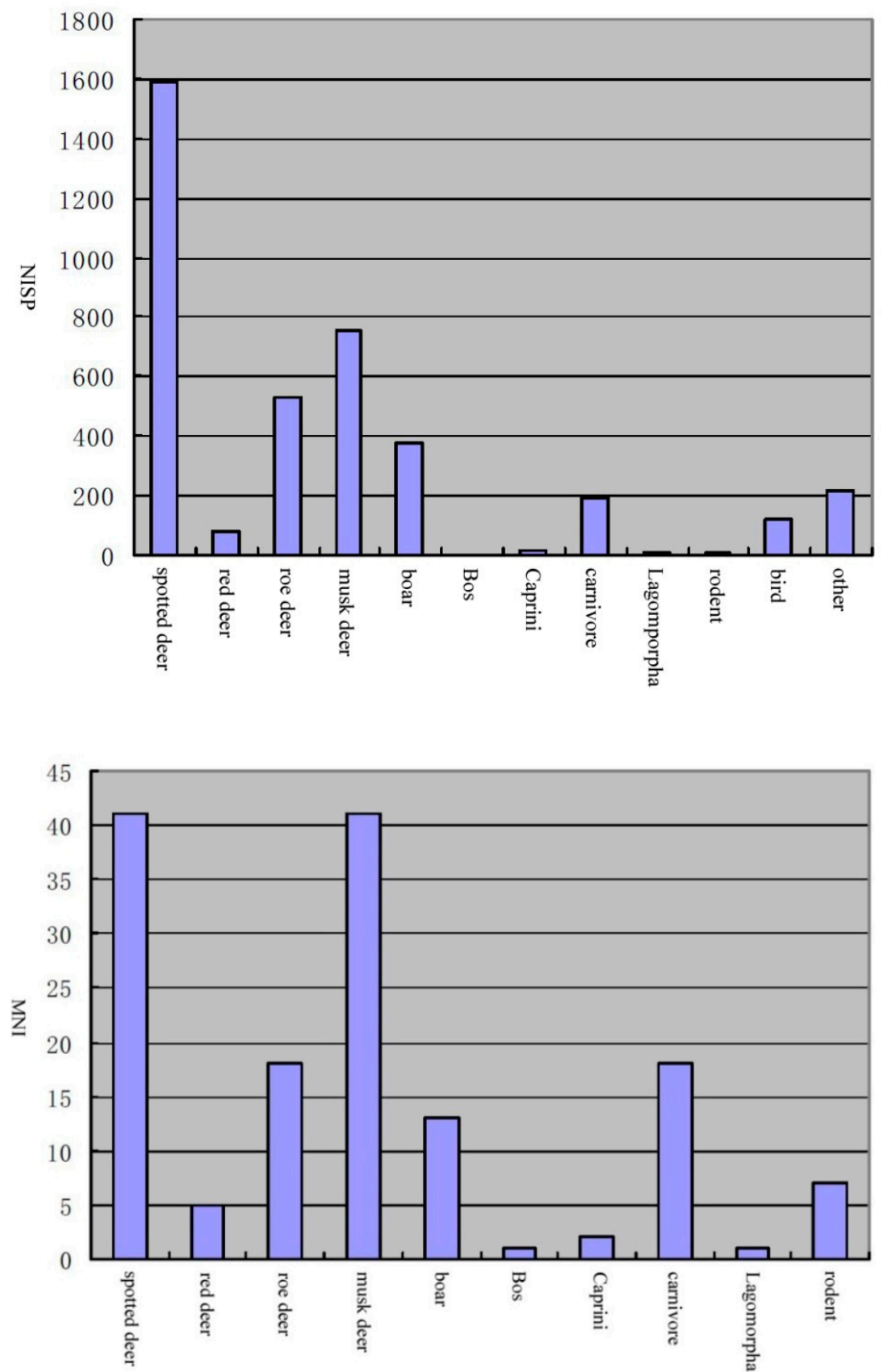

Figure 13. Number of identified specimens (NISPs) and minimal number of individuals (MNIs) of fauna at the Donghulin site (permission from Dr. Tian-Xing Cui).

To sum up, the current published archaeological record is inadequate to explain the cultural process of microblade technology disappearance in northern China. However, the correlation between the decline of microblade-based societies and the rise of agriculture is certain, and we need further detailed studies to build the links.

\section{Conclusions}

This paper does not aim to provide a synthesis of archaeological sites associated with microblade technology, but rather to explore the mechanisms of cultural change for prehistoric hunter-gatherers equipped with microblade products during and after the Last Glacial Maximum. Three sequential 
stages of microblade-based societies are addressed in this paper, and the conclusion can be summarized as follows:

(1) Based on the calculated results in the EnvCalc2.1 program under LGM climatic conditions, especially the variables BIO5 (primary biomass), WDEN (projected density) and EXGRP3 (annual or several-year aggregation size), the plant use at the Shizitan site might not be linked with resource intensification, but rather with social group aggregation in a region with relatively high productivity of plant resources. However, the equifinality in the archaeological explanation needs to be further studied to evaluate alternative hypotheses, including the scale and seasonality of human aggregation and the possibility of resource intensification.

(2) The sites dated to the Pleistocene-Holocene transition experienced Paleolithic to Neolithic technological and economic transitions, marked by use of pottery, appearance of permanent settlements, and widespread adoption of ground stone tools. The broad spectrum revolution and subsistence intensification can be explained within the framework of the macroecological approach; the replacement of the hunting-dominated economy by a gathering-and/or-fishing-dominated economy is linked with population growth during the interglacial or interstadial periods, matching the maps under the packed condition of regional population. Decreased mobility also provided the occupants an opportunity to use low-energy food resources through dietary expansion and/or intensification. Against this background, microblade-based weaponry became less significant in daily life due to the previously open landscape becoming more closed, leading to a decrease in the density of large herd ungulates, and durable ground/polished and expedient stone tools were favored.

(3) The disappearance of microblade technology accompanies the end of microblade-based societies in North China. At present, there are very few publications on this period but the microblade assemblages at the Donghulin site show much less standardization in microblade production. This might be linked with the full adaptation of sedentary lifeways and significant decreases of wild ungulates during the Neolithic.

The present research provides a case study using the macroecological approach for exploring the technological change of prehistoric foraging societies equipped with microblade technology in North China, facing dramatic climatic oscillation during the end of the Ice Age. Although a research framework has been built in this project, more substantial archaeological research is needed using systematic observation of interassemblage variability, a well-controlled chronological database, detailed studies on the function of targeted tools with the aid of use-wear analysis, and interdisciplinary projects on land-use patterns and resource exploitation to test hypotheses proposed here on the cultural dynamics of the microblade-based societies.

Funding: This research received no external funding

Acknowledgments: This paper is developed from the author's dissertation at the Department of Anthropology, University of New Mexico, under the supervision of Lawrence Straus, Bruce Huckell, Robert Hitchcock, Amber Johnson, and Pei-Lin Yu. The research was supported by the Binford Fellowship, TAships, CSC, Moore Research Grant, and the CCKF when I was in the PhD program at the UNM. This project is also supported by the Subject Fundamental Development Project of Fudan University. Yanhua Song and Fagang Wang as well as other archaeologists showed me Paleolithic collections in Shanxi and Hebei Provinces. Tianxing Cui gave me permission to use the archaeological profile as Figure 13 in the present paper. The reviewers provided valuable and constructive suggestions. In addition, this paper is dedicated to Lewis Binford and Ofer Bar-Yosef, who provided me with a toolkit and precious suggestions to deal with the microblade technology issue in prehistory.

Conflicts of Interest: The author declares no conflict of interest.

\section{References}

1. Chen, S.-Q.; Yu, P.-L. Early “Neolithics” of China: Variation and Evolutionary Implications. J. Anthropol. Res. 2017, 73, 149-180. [CrossRef]

2. Chen, S.-Q.; Yu, P.-L. Intensified Foraging and the Roots of Farming in China. Anthropol. Res. 2017, 73, 381-412. [CrossRef] 
3. Binford, L.R. Constructing Frames of Reference: An Analytical Method for Archaeological Theory Building Using Hunter-Gatherer and Environmental Data Sets; University of California Press: Berkeley, CA, USA, 2001.

4. Chen, S.-Q. Adaptive Changes of Hunter-Gatherers during the Late Pleistocene-Early Holocene Transition in China. Acta Anthropol. Sin. 2006, 25, 195-207. (In Chinese)

5. Binford, L.R. Willow smoke and dogs' tails: Hunter-gatherer settlement systems and archaeological site formation. Am. Antiq. 1980, 45, 4-20. [CrossRef]

6. Zhang, M. Explaining Variation and Change among Late Pleistocene and Early Holocene Microblade-Based Societies in Northeastern Asia. Ph.D. Thesis, University of New Mexico, Albuquerque, NM, USA, May 2019.

7. Chen, S.-Q. Prehistoric Modernization: A Cultural Ecological Approach of the Origins of Agriculture; Science Press: Beijing, China, 2014. (In Chinese)

8. Binford's Hunter-Gatherer Data. Available online: http://ajohnson.sites.truman.edu/data-and-program/ (accessed on 20 June 2019).

9. Johnson, A.L. Exploring adaptive variation among hunter-gatherers with Binford's frames of reference. J. Anthropol. Res. 2014, 22, 1-42. [CrossRef]

10. Johnson, A.L.; Collins, C.; Turchin, P.; Cesaretti, R. Global E Regional Frameworks for Comparing Agricultural Intensification \& Productivity Across Cases; SAA: Washington, DC, USA, 2018.

11. Yu, P.-L. Pit Cooking and Intensification of Subsistence in the American Southwest and Pacific Northwest. Ph.D. Thesis, Department of Anthropology, Southern Methodist University, Dallas, TX, USA, July 2006.

12. Yuan, S.-X.; Chen, T.-M.; Zhou, K.-S. Radiocarbon dates and pollen analysis of the Nanzhuangtou site (Appendix of Primary text excavation report of the Nazhuangtou site, Xushui County, Hebei Province). Kaogu 1992, 11, 967-970. (In Chinese)

13. Ren, S.-N.; Wu, Y.-L. Chinese Archaeology: Neolithic; China Social Science Press: Beijing, China, 2010. (In Chinese)

14. Yu, J.-C. Neolithic discoveries and studies of Beijing. In Basheji; Bingwen, Y., Ed.; Beijing Library Press: Beijing, China, 1998; pp. 39-44. (In Chinese)

15. Cui, T.-X. A Study on Lithic Assemblage of the Donghulin Site: Lithic Industry and Human Behaviors in the Paleolithic to Neolithic Transition, Dissertation, School of Archaeology and Museology. Ph.D. Thesis, Peking University, Beijing, China, 2010. (In Chinese).

16. He, J.-N.; Zhang, S.-L.; Wang, S.-Z.; Wang, J.-Y.; Zhao, J.-F.; Gao, X.-X.; Wang, Y.-P. Archaeological report of Lijiagou site (north area), Xinmi, Henan Province (2009). Anc. Civiliz. 2013, 9, 177-207. (In Chinese)

17. Zhao, J.-F.; Zhang, S.-L.; Wang, S.-Z.; He, J.-N.; Gao, X.-X.; Wang, J.-Y.; Wang, Y.-P. Archaeological report of Lijiagou site (south area), Xinmi, Henan Province (2009). Anc. Civiliz. 2013, 9, 208-239. (In Chinese)

18. Sun, B.; Wagner, M.; Zhao, Z.-J.; Li, G.; Wu, X.-H.; Tarasov, P.E. Archaeological discovery and research at Bianbiandong early Neolithic cave site, Shandong, China. Quat. Int. 2014, 348, 169-182. [CrossRef]

19. Li, W.-C.; Song, G.-D.; Wu, Y. Preliminary analysis of starch grains on the surface of stone artifacts from the Kengnan site. Acta Anthr. Sin. 2014, 33, 70-81. (In Chinese)

20. Mei, H.-J. Transition from Paleolithic to Neolithic in the Nihewan Basin: A Study of the Discoveries from the Yujiagou Site. Ph.D. Thesis, College of Archaeology and Museology, Peking University, Beijing, China, 2007. (In Chinese).

21. IA-CASS. Radiocarbon Dates in Chinese Archaeology, 1965-1991; Institute of Archaeology, Ed.; Wenwu Press (Cultural Relics Publishing House): Beijing, China, 1991. (In Chinese)

22. Nagatomo, T.; Shitaoka, Y.; Namioka, H.; Sagawa, M.; Wei, Q. OSL dating of the strata at Paleolithic sites in the Nihewan Basin, China. Acta Anthr. Sin. 2009, 28, 276-284. (In Chinese)

23. Li, Z.-Y.; Xing, Y.; Mu, J.-H.; Wu, X.-L.; Li, Y.-N.; Kato, S. Excavation report of microliths in Layer 5 at the Lingjing Xuchang Man site, 2008-2013. Huaxia Archaeol. 2018, 2, 3-33. (In Chinese)

24. Song, Y.-H. Study on the Quartzite Artifacts in Shizitan Site, Jixian, Shanxi Province. Ph.D. Thesis, University of Chinese Academy of Sciences, Beijing, China, 2011. (In Chinese).

25. Song, Y.-H.; Shi, J.-M. A preliminary excavation report of the Locality S29, Shizitan site, Ji County, Shanxi Province. Kaogu 2017, 2, 35-51. (In Chinese)

26. Song, Y.-H.; Shi, J.-M. Preliminary excavation report of Shizitan Site, Locality S5, Ji County, Shanxi Province. Kaogu 2016, 4, 3-15. (In Chinese)

27. Liu, D.-C.; Chen, F.-Y.; Zhang, X.-L.; Pei, S.-W.; Gao, X.; Xia, Z.-K. Preliminary comments on the paleoenvironment of the Shuidonggou Locality 12. Acta Anthropol. Sin. 2008, 27, 295-303. (In Chinese) 
28. Yi, M.-J. Adaptive Strategies of Hunter-Gatherers during the Late Upper Paleolithic in Northern China: An Archaeological Research on the Shuidonggou 12. Ph.D. Thesis, Institute of Vertebrate Paleontology and Paleoanthropology, University of Chinese Academy of Sciences, Beijing, China, 2012. (In Chinese).

29. Elston, R.G.; Xu, C.; Madsen, D.B.; Zhong, K.; Bettinger, R.L.; Li, J.-Z.; Brantingham, P.J.; Wang, H.M.; Yu, J. New dates for the north China Mesolithic. Antiquity 1997, 71, 985-993. [CrossRef]

30. Barton, L.; Brantingham, P.J.; Ji, D.-X. Late Pleistocene climate change and Paleolithic cultural evolution in northern Chinanorthern China: Implications from the Last Glacial Maximum. Dev. Quat. Sci. 2007, 9, 105-128.

31. Liu, L.; Levin, M.J.; Bonomo, M.F.; Wang, J.-J.; Shi, J.-M.; Chen, X.-C.; Han, J.-Y.; Song, Y.-H. Harvesting and processing wild cereals in the Upper Palaeolithic Yellow River Valley, China. Antiquity 2018, 92, 603-619. [CrossRef]

32. Liu, L.; Bestel, S.; Shi, J.-M.; Song, Y.-H.; Chen, X.-C. Paleolithic human exploitation of plant foods during the last glacial maximum in North China. Proc. Natl. Acad. Sci. USA 2013, 110, 5380-5385. [CrossRef]

33. Liu, L.; Ge, W.; Bestel, S.; Jones, D.; Shi, J.-M.; Song, Y.-H.; Chen, X.-C. Plant exploitation of the last foragers at Shizitan in the Middle Yellow River Valley China: Evidence from grinding stones. J. Archaeol. Sci. 2011, 38, 3524-3532. [CrossRef]

34. Straus, L.G.; Goebel, T. Humans and Younger Dryas: Dead end, short detour, or open road to the Holocene? Quat. Int. 2011, 242, 259-261. [CrossRef]

35. Straus, L.G. The archaeology of the Pleistocene-Holocene transition in Southwest Europe. In Humans at the End of the Ice Age: The Archaeology of the Pleistocene-Holocene Transition; Straus, L.G., Eriksen, B.V., Erlandson, J.M., Yesner, D.R., Eds.; Plenum Press: New York, NY, USA; London, UK, 1996; pp. 83-99.

36. Binford, L.R. In Pursuit of the Past: Decoding the Archaeological Record; Thames and Hudson London: New York, NY, USA, 1983.

37. Binford, L.R. Post-Pleistocene adaptation. In New Perspectives in Archeology; Binford, S.R., Binford, L.R., Eds.; Aldine Publ. Co.: Chicago, IL, USA, 1968; pp. 313-341.

38. Zhang, M. Microblade-based Societies: A new perspective on microblade technology in northern Chinanorthern China after the Last Glacial Maximum. Acta Archaeol. Sin. 2020. in press (In Chinese)

39. Andrefsky, W. Raw-material availability and the organization of technology. Am. Antiq. 1994, 59, 21-34. [CrossRef]

40. Nelson, M.C. The study of technological organization. Archaeol. Method Theory 1991, 3, 57-100.

41. Zhu, Z.-Y.; Gao, X. A study of wedge-shaped cores from Hutouliang site. Acta Anthropol. Sin. 2006, 25, 129-142. (In Chinese)

42. Li, Z.-Y.; Li, Y.-N.; Kato, S. Observations of microblade core technologies from Level 5 of the Xuchang Man site, Lingjing. Acta Anthr. Sin. 2014, 33, 285-303. (In Chinese)

43. Yi, M.-J.; Gao, X.; Wang, H.-M.; Pei, S.-W.; Chen, F.-Y. A Study on Cores Unearthed from the Shuidonggou Locality 12 in 2007. Acta Anthr. Sin. 2015, 34, 166-179. (In Chinese)

(C) 2020 by the author. Licensee MDPI, Basel, Switzerland. This article is an open access article distributed under the terms and conditions of the Creative Commons Attribution (CC BY) license (http://creativecommons.org/licenses/by/4.0/). 